\title{
On the quark scaling theorem and the polarisable dipole of the quark in a scalar field
}

\author{
Engel Roza \\ Philips Research Labs, Eindhoven, The Netherlands (retired) \\ Email: engel.roza@onsbrabantnet.nl
}

\section{Summary}

In this article the possible impact on the present state of particle physics theory is discussed of two unrecognized theoretical elements. These elements are the awareness that (a) the quark is a Dirac particle with a polarisable dipole moment in a scalar field and that (b) Dirac's wave equation for fermions, if derived from Einstein's geodesic equation, reveals a scaling theorem for quarks. It is shown that recognition of these elements proves by theory quite some relationships that are up to now only empirically assessed, such as for instance, the mass relationships between the elementary quarks, the relationship between the bare mass and the constituent mass of quarks, the mass spectrum of hadrons and the mass values of the $Z$ boson and the Higgs boson.

Keywords: polarisable Dirac dipole; quark scaling; hadron mass spectrum; Z boson; Higgs boson

\section{Introduction}

This article is aimed to discuss the possible impact that the recent discovery of a third-type Dirac particle (next to the electron-type and the Majorana-type), might have had on the development of particle physics theory, were it would have been revealed quite some decades ago. The discussion will be focussed on the bonds between quarks in mesons and baryons. As is well-known, canonical theory is captured in a rather abstract mathematical formalism. This formalism has been developed under adoption of some axiomatic attributes that were unknown prior to the development of the Standard model. Among these are, for instance, weak isospin and hypercharge. They show up as quantum numbers attributed to the elementary fermions [1], such as listed in Table I.

Table I

\begin{tabular}{|l|l|l|l|l|}
\hline Fermions & $\mathrm{u}$ & $\mathrm{d}$ & $\mathrm{e}^{-}$ & $v_{e}$ \\
\hline $\mathrm{S}$ - spin & $1 / 2$ & $1 / 2$ & $1 / 2$ & $1 / 2$ \\
\hline $\mathrm{m}_{0}$-mass & $?$ & $?$ & $m_{e}$ & $\approx 0$ \\
\hline$I_{Z}$-weak isospin charge & $1 / 2$ & $-1 / 2$ & $-1 / 2$ & $1 / 2$ \\
\hline $\mathrm{Y}$-hypercharge & $1 / 3$ & $1 / 3$ & -1 & -1 \\
\hline$Q=I_{Z}+Y / 2$ & $2 / 3$ & $-1 / 3$ & -1 & 0 \\
\hline
\end{tabular}

It is my aim to show how these attributes are related to those of quark that has a polarisable dipole moment in a scalar potential field. Such a dipole moment is a unique property of 
Dirac's third type, while it is absent in electron-type ones. As will be shown, this dipole moment is also the key for assigning reliable figures to the rest masses of elementary quarks and their hadron composites. It will be shown that a re-interpretation of these attributes removes the reason to accept the asymmetrical electric charge break of quarks. It will be shown as well that the number of elementary fermions can be reduced significantly.

Like all elementary fermions, quarks follow Fermi-Dirac statistics, obey the Pauli exclusion principle, have half integer spin and have distinct antiparticles. They can be modelled with the Dirac equation. The canonic formulation of Dirac's particle equation reads as $[2,3]$,

$\left(i \hbar \gamma^{\mu} \partial_{\mu} \psi-\beta m_{0} c \psi\right)=0$

where $\beta$ is a $4 \times 4$ unity matrix and where the $4 \times 4$ gamma matrices have the properties,

$\gamma_{\mu} \gamma_{v}+\gamma_{v} \gamma_{\mu}=0$ if $\mu \neq v$; and $\gamma_{0}^{2}=1 ; \gamma_{i}^{2}=-1$

As usual, $c$ is the vacuum light velocity, $\hbar$ is the reduced Planck constant and $m_{0}$ is the rest mass of the particle. While this equation captures a basic attribute as mass and attributes as spin state and particle/antiparticle state, it does not include quite some other properties of elementary fermions. It does not even include electric charge as attribute, while Dirac's theory is originally conceived for electrons. It includes mass $m_{0}$ and nuclear spin $S$, but the hypercharge and weak isospin are missing. These are rather artificial attributes, conceived in a mathematical model in which empirical phenomena are captured by axiomatic abstraction.

While spin $S$ can be physically understood in terms of the eigen value of an elementary angular moment $\hbar$, weak isospin has no known physical interpretation. Apart from its relationship with the electric charge as shown by the Gell-Mann-Nishijma formula [4,5] at the bottom line of Table I, it plays a role in the classification of hadrons, in interactions between nuclear particles and in the interaction with the omni-present energetic background field, known as the Higgs field. Weak isospin shows the same behaviour as the nuclear spin $S$ in the sense of being subject to the same algebra rules as nuclear spin, thereby establishing an isospin triplet state $|1, \pm 1\rangle$ or $|1,0\rangle$ next to a singlet state $|0,0\rangle$.

Particle physics theory has been developed over many decades of years. As is well-known, a major milestone in this development was set in 1961 by Gell-Mann and Ne'eman, dubbed as the Eightfold Way [6]. It is a classification scheme for hadrons, in which isospin has been introduced as a heuristic attribute without a known physical interpretation. One may wonder how this scheme would have been set up if isospin would have been comprehended physically. Within the scope of this article, it is my aim to show that such a physical interpretation allows a less heuristic alternative for the Eightfold Way.

To show that such a novel view might be a useful complement to present theory, some problems will be addressed that are difficult to solve with present-state theory. Examples of 
such problems are mass related, because present theory shows a weakness in that respect. In particular, it will be shown how the mass spectrum of hadrons can be calculated to a rather high precision, how the masses of the bare quarks can be calculated and why, for instance, the mass of a neutral pion is $4.6 \mathrm{MeV}$ larger than that of a charged pion.

After a summary in chapter 2 of the third-type Dirac particle, the quark will be profiled in relationship with the electroweak theory. Thereafter, in chapters 4 and 5 the archetype meson (pion), based on this profile, will be described thereby showing the relationship between the constituent mass of quarks and their bare masses. Chapter 6 contains a numerical assessment of the $\mathrm{Z}$ boson mass. In chapter 7 a description is given of the quarkscaling theorem, followed in chapter 8 by an assessment of the Higgs boson mass. Chapter 9 deals with the influence of electromagnetic interaction in mesons. In chapter 10, a short description of baryons is given on the basis of the developed theory. Chapter 11 contains a discussion and the conclusion. In these chapters, quite some results are invoked from previously documented works in publications and preprints. The highlight on the third-type Dirac particle and the quark-scaling theorem as two unrecognized theoretical principles will place those in a better context.

\section{Summary of the third}

Where the canonical set of gamma matrices is given by,

$$
\gamma_{0}=\left[\begin{array}{cc}
I & 0 \\
0 & -I
\end{array}\right] ; \gamma_{1}=\left[\begin{array}{cc}
0 & \sigma_{1} \\
-\sigma_{1} & 0
\end{array}\right] ; \gamma_{2}=\left[\begin{array}{cc}
0 & \sigma_{2} \\
-\sigma_{2} & 0
\end{array}\right] ; \gamma_{3}=\left[\begin{array}{cc}
0 & \sigma_{3} \\
-\sigma_{3} & 0
\end{array}\right] ; \beta=\left[\begin{array}{cc}
I & 0 \\
0 & I
\end{array}\right],
$$

the $\gamma$-set of the third type has been found as,

$$
\gamma_{0}=\left[\begin{array}{cc}
0 & -I \\
-I & 0
\end{array}\right] ; \gamma_{1}=\left[\begin{array}{cc}
\mathrm{i} \sigma_{1} & 0 \\
0 & -\mathrm{i} \sigma_{1}
\end{array}\right] ; \gamma_{2}=\left[\begin{array}{cc}
\mathrm{i} \sigma_{2} & 0 \\
0 & -\mathrm{i} \sigma_{2}
\end{array}\right] ; \gamma_{3}=\left[\begin{array}{cc}
\mathrm{i} \sigma_{3} & 0 \\
0 & -\mathrm{i} \sigma_{3}
\end{array}\right] ; \beta=\left[\begin{array}{cc}
0 & I \\
-I & 0
\end{array}\right] \text {, }
$$

where $\sigma_{i}$ are the Pauli matrices.

Although the wave equation of the electron type and that of the "third" are hardly different, there is a major difference in an important property. Both have two dipole moments. A first one, to be indicated in this text as the angular dipole moment, is associated with the elementary angular momentum $\hbar$. The second one, to be indicated as the polarisable dipole moment is associated with the vector $\hbar / c$. These dipole moments show up in the calculation of the excess energy of the particle in motion subject to a vector potential $A\left(A_{0}, A_{x}, A_{y}, A_{z}\right)$. In the canonic case (2a) we have,

$$
\Delta E=\frac{e \hbar}{2 m_{0}}\left[\begin{array}{cc}
\bar{\sigma} \cdot \mathbf{B} & 0 \\
0 & \bar{\sigma} \cdot \mathbf{B}
\end{array}\right]+\frac{e \hbar}{2 m_{0} c}\left[\begin{array}{cc}
0 & \mathrm{i} \bar{\sigma} \cdot \mathbf{E} \\
\mathrm{i} \bar{\sigma} \cdot \mathbf{E} & 0
\end{array}\right]
$$


where $\bar{\sigma}$ is the Pauli vector, defined by

$$
\bar{\sigma}=\sigma_{1} \mathbf{i}+\sigma_{2} \mathbf{j}+\sigma_{3} \mathbf{k}
$$

where $(\mathbf{i}, \mathbf{j}, \mathbf{k})$ are the spatial unit vectors and where $\mathbf{B}$ and $\mathbf{E}$ are generic field vectors derived from the vector potential. The redundancy in (3) allows writing it as,

$$
\Delta E=\frac{e}{2 m_{0}}(\bar{\sigma} \boldsymbol{\hbar} \cdot \mathbf{B}+\mathrm{i} \bar{\sigma} \boldsymbol{\hbar} / \boldsymbol{c} \cdot \mathbf{E})
$$

The electron has a real first dipole moment $\left(e \hbar / 2 m_{0}\right)$, known as the magnetic dipole moment, and an imaginary second dipole moment (ieh/2 $m_{0} c$ ), known as the anomalous electric dipole moment. The spin vector $\boldsymbol{S}=\bar{\sigma} / 2$ has an eigen value $|\boldsymbol{S}|=1 / 2$. In the case that the Dirac particle is of the third type as defined by (2b), we have [7],

$$
\Delta E=\frac{e}{2 m_{0}}(\bar{\sigma} \boldsymbol{\hbar} \cdot \mathbf{B} \pm \bar{\sigma} \hbar / \mathbf{c} \cdot \mathbf{E})
$$

The generic third type Dirac particle has two real dipole moments, generically, i.e., without identifying it as an electromagnetic one, to the amounts of $\bar{\sigma} \hbar$, respectively $\bar{\sigma} \hbar / c$. If the quark would be of the electron type, it would not be polarisable in a scalar potential field, because such a field is Coulomb-like and is unable to polarize an imaginary electric dipole moment. If, however, the quark is a third type, its second dipole moment can be polarized under influence of a scalar potential field. This field is not necessarily the electromagnetic one. The coupling factor is not necessarily the elementary electric charge. If the field is just a static one, eq. (5) can be written as,

$$
\Delta E=-\frac{g \bar{\sigma}}{2 m_{0}}\left(\hbar / c \cdot \nabla A_{0}\right),
$$

where $g$ is a generic coupling factor. Hence, taking into account that the eigen value of the spin vector with the state variable $\bar{\sigma}$ is $|\boldsymbol{S}|=|\bar{\sigma} / 2|=1 / 2$, the dipole moment $p$ of a quark particle in a scalar nuclear field $\nabla . A_{0}$ is given by,

$$
p=\frac{g}{m_{0}} \frac{\hbar}{2 c} .
$$

Summarizing: conceiving the quark as a third type Dirac particle allows to consider the quark as a particle that, under influence of its dipole moment, may "spin" (i.e., can be polarized) in a scalar nuclear potential field. The associated quantity $\bar{\sigma}$ is a state variable that can be conceived as isospin next to the nuclear spin associated with the elementary angular momentum $\hbar$. 


\section{A qualitative profile of a quark and an antiquark bond.}

Before profiling the quark in mathematical terms, it might be useful to start with a qualitative description on the consequences that this polarisable dipole moment might have.

Similarly as an electron, the quark has an energetic monopole. For an electron, the monopole is an electric point charge. For the quark it is the nuclear equivalent of the electric point charge. Next to the monopole, the electron and the quark have two dipole moments. These dipole moments are the results from the elementary angular momentum $\hbar$ and the elementary mass dipole moment $\boldsymbol{\hbar} / \boldsymbol{c}$. In the case of an electron, these dipole moments give rise to, respectively, a real magnetic dipole and an imaginary electric dipole. In the case of a quark, these dipole moments gives rise to, respectively, a real equivalent of the magnetic dipole and a real nuclear equivalent of the electric dipole. While, due to its imaginary value, the electric dipole moment of the electron cannot be polarized in a scalar potential field, the nuclear equivalent can, because of its real value.

Similarly as the monopole of the electron, the monopole of the quark, spreads a scalar potential field. This field is able to polarize the electric dipole equivalent of another quark. Such a dipole spreads an en energetic potential with $x^{-2}$ dependency along the orientation axis of the dipole. As a consequence, an equilibrium of forces can arise between a repelling force from the $r^{-1}$ monopole field dependency and the attractive force with $x^{-2}$ dipole field dependency from suitably aligned dipoles of two quarks. Because nuclear forces have a short range, these potential fields must experience a shielding effect akin to the shielding of the field of an electric point charge in an ionized plasma. This shielding is known as the Debije effect. It occurs under influence of an omni-present fluidal field of energy, in particle physics known as the Higgs field, in gravity known as the cosmological background field due to the Cosmological Constant. Hence, in qualitative terms, the potential field of a quark along the axis of the polarisable dipole, can be expressed as,

$\Phi(\lambda x)=\Phi_{0} \exp (-\lambda x)\left\{\frac{1}{(\lambda x)^{2}}-w \frac{1}{\lambda x}\right\}$

where $\lambda$ ( in dimension $\mathrm{m}^{-1}$ ) is a measure for the range of the nuclear potential, where $\Phi_{0}$ (in units of energy, i.e. joule) is a measure for one of the two quark's "charges", and where $w$ is a dimensionless weigh factor that relates the strength of the monopole field to the dipole field. The far field, decaying as $\exp (-\lambda x) / \lambda x$ is due to the monopole. It can be seen as the weak interaction between the quarks. The near field, decaying as $\exp (-\lambda x) /(\lambda x)^{2}$ can be seen as the strong interaction between the quarks. The strong interaction is due the polarisable dipole. Because of the lack of this dipole, the electron and the other leptons are not subject to strong interaction. Hence, unlike quark bonds, such lepton bonds don't exist. Figure 1 shows a schematic configuration between two elementary quarks.

Let us proceed by considering this assembly as a bond between a quark and an antiquark. 
Note: Although the considerations between eqs. (10) and (44) to be described next, can be found in leading textbooks $[1,10]$, the summary is required for proper understanding of the role of Dirac's polarisable dipole.

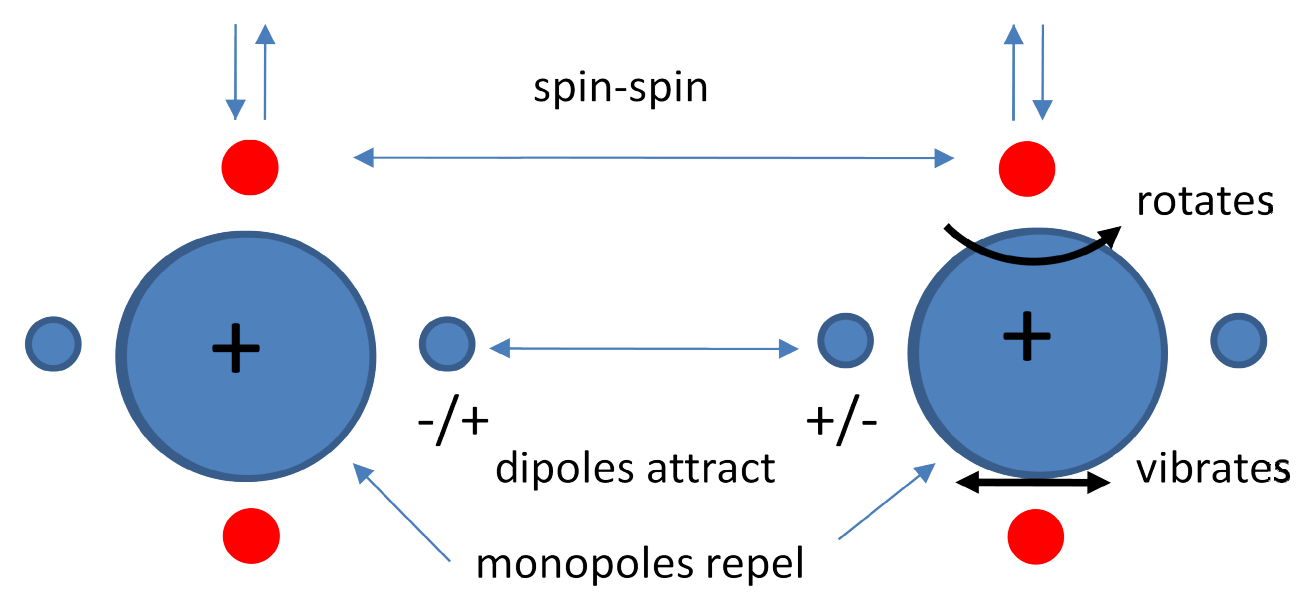

Fig. 1. A quark has two real dipole moments, hence two dipoles. One of these (horizontally visualized) is polarisable in a scalar potential field. The other one (vertically visualized) is not. The dipole moments are subject to spin statistics. However, the polarity of the horizontal one is restrained by the bond: the horizontal dipoles are only oriented in the same direction: either inward to the centre or outward from the centre.

In canonic theory the interaction between nuclear particles is described in terms of fields. The field of a particle can be viewed as the mapping of its momenta $p_{\mu}$ on the amplitudes $\Psi_{\mu}$ of the four components of the solution of Dirac's equation, i.e., as

$\left\{p_{0}, p_{1}, p_{2}, p_{3}\right\} \rightarrow\left\{\Psi_{0}, \Psi_{1} \Psi_{2}, \Psi_{3}\right\}$

This mapping is visualized in figure 1 for $1+2$ dimensionality.

The left-hand part is a geometric interpretation of Einstein's energy law in Hawking metric $(+,+,+,+)$ for $(\mathrm{i} c t, x, y, z), \mathrm{i}=\sqrt{-1}$. The well-known Einsteinean energy expression of a generic free moving particle with rest mass $m_{0}$ is given as,

$$
E_{W}=\sqrt{\left(m_{0} c^{2}\right)^{2}+(c|\mathbf{p}|)^{2}}
$$

where $\mathbf{p}$ is the three-vector momentum $(\mathrm{d} s / \mathrm{d} t$, not be confused with the fourvector momentum $\boldsymbol{p}$ ). Under adoption of the Hawking metric (like, for instance, adopted by Perkins [8]),

$$
E_{W}^{2}=-p_{00}^{2} c^{2}=\left(m_{0} c^{2}\right)^{2}+c^{2} p_{1}^{2}+c^{2} p_{2}^{2}+c^{2} p_{3}^{2},
$$

which can be normalized as, 


$$
p_{00}^{\prime 2}+p_{1}^{\prime 2}+p_{2}^{\prime 2}+p_{3}^{\prime 2}+1=0 ; \quad p_{\mu}^{\prime}=\frac{p_{\mu}}{m_{0} c}
$$

This allows representing the momentum space of a moving particle in free space as a sphere with unit radius.

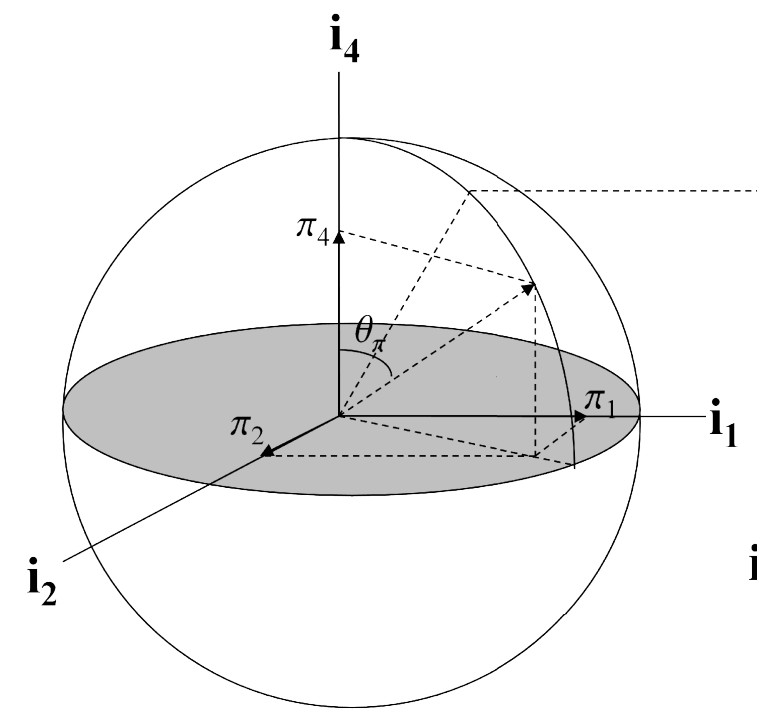

momentum space

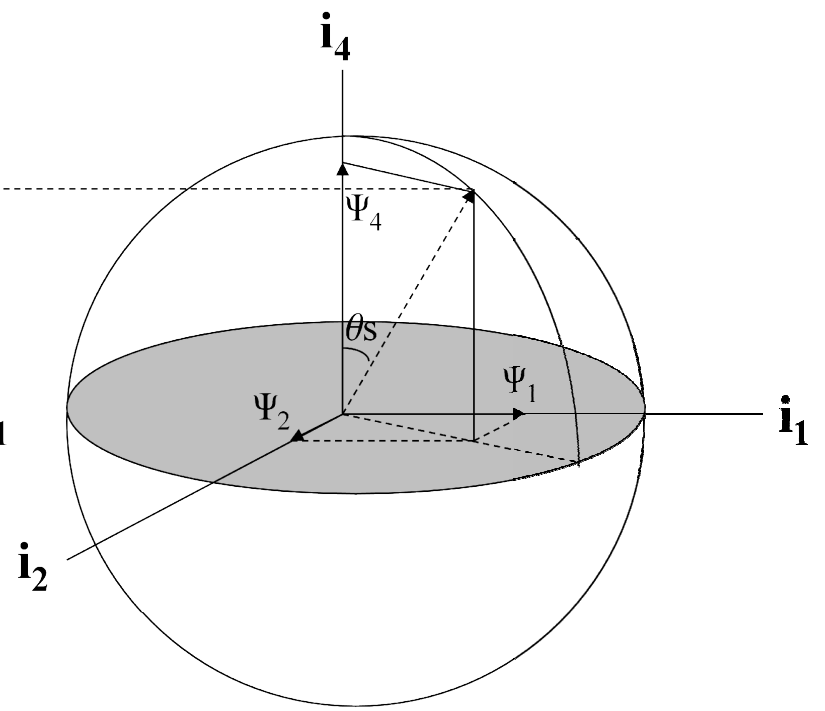

spinor space

Fig. 2. A visual interpretation of the mapping of the particle's momenta into amplitudes of Dirac's wave function solution. Note that this mapping is not 1 to 1 .

The right-hand part is a geometric interpretation of the absolute values of the amplitudes $\Psi_{\mu}$ of the four components of the solution of Dirac's equation. The amplitudes themselves are complex quantities. As a consequence of the semantics of the particle's wave function, these amplitudes can be represented as orthogonal vectors in a unit sphere. Note that this mapping is not one-to-one. In momentum space, the angle $\vartheta_{\pi}$ between the temporal momentum $p_{0}$ and the vector sum of the spatial momenta $p_{i}$ is a global invariant. As long as the particle's energy is not changed by a field of force, the angle remains the same. In spinor space, there is a characteristic angle $\vartheta_{s}$ between the component $\Psi_{0}$ associated with the temporal momentum and the vector sum of the components $\Psi_{i}$ associated with the spatial momenta. Although the mapping is not one-to one, the angle $\vartheta_{s}$ is globally invariant. Under influence of forces on the momenta, the angle $\vartheta_{\pi}$ will change, while the radius of the momentum space will remain the same owing to the normalization. The angle in the spinor space will change as well and the radius of the spinor space will remain the same because of the wave function semantics. These angles play a role in the modification of Dirac's free space equation into a covariant one. By definition, the covariant equation, valid for particles moving in a field of forces has the same format as the free space equation after redefining the normal differential operators into covariant ones, i.e. $\partial_{\mu} \rightarrow D_{\mu}$. The prescription how to do it, is the modification of a global invariant quantity into a local invariant one. By modifying the global invariance of the Lorentz transform into a local invariant one, Einstein 
has been able to derive the transformation rule for the covariant derivatives that modified his equations of Special Relativity in free space into covariant equivalents for his equations of General Relativity. Paul Dirac's prescription for making his equation (1) covariant in a conservative field of forces $\boldsymbol{A}\left(A_{0}, \mathbf{A}\right)$,

$p_{\mu}^{\prime} \rightarrow p_{\mu}^{\prime}+g A_{\mu}^{\prime} \quad$ and $\quad p_{\mu}^{\prime} \rightarrow \hat{p}_{\mu} \psi+g A_{\mu}^{\prime} \psi ; \hat{p}_{\mu}^{\prime}=\frac{1}{m_{0} c} \frac{\hbar}{\mathrm{i}} \frac{\partial}{\partial x_{\mu}}$

can be interpreted as the modification of the global invariance of $\vartheta_{\pi}$ and $\vartheta_{s}$ into local invariant ones, because (14) are just infinitesimal rotations in, respectively, momentum space and spinor space. Effectively, these rotations takes place in 2D space, as long as a single particle is involved.

It is instructive to consider the particle's antiparticle in this picture. The momentum amplitude of the antiparticle has the same value as the amplitude of the vector sum of the spatial momenta of the particle. And, in spite of the fact that the mapping from momenta to the values of the spinor components is non one-to-one, the absolute value of the amplitude of the spinor component associated with the temporal momentum is equal to the value of the vector sum in spinor space of the amplitudes of the spinor components associated with the spatial momenta of the antiparticle.

This picture allows to represent the particle-antiparticle bond as a $2 \times 2$ matrix $\Psi_{p a}$,

$\Psi_{p a}=\left[\begin{array}{ll}\Psi_{1 t} & \Psi_{1 s} \\ \Psi_{2 t} & \Psi_{2 s}\end{array}\right]$

where $\Psi_{i t}$ represent the components associated with the temporal momenta and where $\Psi_{i s}$ represent those associated with the spatial momenta. This matrix now has the following properties,

$$
\Psi_{1 t} \Psi_{1 t}^{*}+\Psi_{1 s} \Psi_{1 s}^{*}=1 ; \Psi_{2 t} \Psi_{2 t}^{*}+\Psi_{2 s} \Psi_{2 s}^{*}=1 ; \Psi_{2 s}=\Psi_{1 t}^{*} \text { and } \Psi_{2 t}=\Psi_{1 s}^{*}
$$

Because of this relationship, the matrix (15) is unitary, i.e.

$\Psi_{p a} \Psi_{p a}^{T}=1$,

where $\Psi_{p a}^{T}$ is the transpose conjugate of $\Psi_{p a}$.

Note that the elements of $\Psi_{p a}$ are complex numbers. All objects that can be represented by such a $2 \times 2$ unitary matrix form a class that is known as the SU(2) Lie-group. It will be clear that a particle-antiparticle bond, consisting of an archetype quark and its antiquark, i.e., the $u$ quark and the $\bar{u}$ quark, will belong to an $\mathrm{SU}(2)$ Group. This even holds for mixed compositions, such as for instance $u$ and $\bar{s}$, because as will be shown later, the strange quark $s$ is a scaled version of the up quark $u$ in the sense of just a difference of their bare 
quark rest masses. Hence the two quark flavors behave the same in the normalized momentum space.

The particle-antiparticle bond can be visualized in the spinor space as an assembly of two vectors that have some position with respect to each other. This position is characterized by three (Euler) angles between the two vectors. As long as the interaction forces that bind the two particles do not change, the three angles can be seen as variables that are globally invariant. An increase or decrease of the interaction force will change the value of the three angles. Because, there are three angles involved, rather than a single one as in the case of a single particle, there might be three independent boson vector fields that may change the relative positions of the two particles in the bond. In that respect, any operation that results in the same Lagrangian condition is equivalent. In other words: it leaves the two particles in the same mathematical group. This gives the recipe for defining a covariant derivative, formally dubbed as gauge, in Dirac-type wave equations of particle bonds. The gauge for particle bonds with a wave function (= field) that is subject to the unitary constraint, is originally generically formulated by Yang and Mills [9]. Let us apply these considerations on the $\mathrm{SU}(2)$ group of the particle-antiparticle such as composed by the quark and antiquark.

A complex $n \times n$ matrix has $2 n^{2}$ real parameters. The unitary condition on the rows removes $n^{2}$ of these and an additional one is removed by the constraint of unit determinant. That leaves 3 degrees of freedom for the SU(2) operator. The matrix (15) can then be generically represented as,

$\left[\begin{array}{cc}\mathrm{e}^{i \beta} \cos \alpha & \mathrm{e}^{i \gamma} \sin \alpha \\ -\mathrm{e}^{-i \gamma} \sin \alpha & \mathrm{e}^{-\mathrm{i} \beta} \cos \alpha\end{array}\right]$

Obviously, this matrix is unitary, thereby meeting the constraints as imposed by (15). Liegroup theory states that any matrix multiplication with the generic SU(2) format as defined in (17) leaves the object in the group. Hence, the transformation that maintains the desired property of Lagrangian equivalence is given by

$$
\Psi \rightarrow \Psi \exp (\mathrm{i} \bar{\sigma} \bar{\vartheta}) \text { with } \bar{\vartheta}=\bar{\vartheta}\left(\vartheta_{1}, \vartheta_{2}, \vartheta_{3}\right) \text { and } \bar{\sigma}=\bar{\sigma}\left(\sigma_{1}, \sigma_{2}, \sigma_{3}\right)
$$

as long as the matrices $\bar{\sigma}=\bar{\sigma}\left(\sigma_{1}, \sigma_{2}, \sigma_{3}\right)$ match with (17). The most simple ones are the three Pauli matrices,

$\sigma_{1}=\left[\begin{array}{cc}0 & 1 \\ 1 & 0\end{array}\right] ; \sigma_{2}=\left[\begin{array}{cc}0 & -\mathrm{i} \\ \mathrm{i} & 0\end{array}\right] ; \quad \sigma_{3}=\left[\begin{array}{cc}1 & 0 \\ 0 & -1\end{array}\right]$

From (19) should follow $D_{\mu} \Psi \rightarrow D_{\mu} \Psi \exp (\mathrm{i} \overline{\overline{ }} \bar{\vartheta})$. This is true if

$D_{\mu} \Psi=\partial_{\mu} \Psi-\mathrm{i} \Psi \partial_{\mu}(\bar{\sigma} \bar{\vartheta})$.

By identifying 
$\bar{\sigma} \bar{\vartheta}=\sigma_{k} \vartheta^{k}=g \sigma_{k} W^{k}$

where $g$ is a generic dimensionless coupling factor,

we get,

$D_{\mu} \Psi=\partial_{\mu} \Psi-\mathrm{ig} \sigma_{k} W_{\mu}^{k} ; \quad k=1,2,3$.

Because $\sigma_{k} W_{k}$ are operations in the field domain with a complex number type, $W_{k}$ cannot be identified as mappings of real valued momenta. Hence, it makes sense to redefine,

$W_{1}^{+}=W_{1}+\mathrm{i} W_{2} ; W_{1}^{-}=W_{1}-\mathrm{i} W_{2} ; W^{0}=W_{3}$.

From (22) and (23),

$D_{\mu} \Psi=\partial_{\mu} \Psi+\operatorname{ig}\left(\tau_{1} W_{\mu}^{+}+\tau_{2} W_{\mu}^{-}+\tau_{0} W_{\mu}^{0}\right)$,

where $\tau_{k}$ now are real valued matrices,

$\tau_{1}=\left[\begin{array}{ll}1 & 0 \\ 0 & 0\end{array}\right] ; \tau_{2}=\left[\begin{array}{cc}0 & 1 \\ 0 & 0\end{array}\right] ; \quad \tau_{0}=\left[\begin{array}{cc}1 & 0 \\ 0 & -1\end{array}\right]$

Note that as yet the + sign and the -sign have no electrical meaning here. The next issue to be solved is the question how to generalize the covariant derivative to an expression that incorporates, next to the weak interaction, the electromagnetic behavior and the strong interaction of the quark as well. Naively, one might suppose that the quark is a monopole for weak interaction energy, electromagnetic energy and strong interaction energy. The canonical electroweak theory unifies electroweak interaction and electromagnetic on the basis of the assumption that the quark is a monopole for electric charge. It leaves strong interaction for an issue to be developed later. A naïve approach to unify weak interaction with electromagnetic interaction would suggest extending the covariant derivative by an additional term, such that

$D_{\mu} \Psi=\partial_{\mu} \Psi+\mathrm{i} g\left(\tau_{1} W_{\mu}^{+}+\tau_{2} W_{\mu}^{-}+\tau_{0} W_{\mu}^{0}\right)+\mathrm{i} g_{e} A_{\mu}$

One might even go a step further, supposing that similarly as $W_{\mu}^{+}$and $W_{\mu}^{-}$are intimately related, $W_{\mu}^{z}$ and $A_{\mu}$ would be related as well. This marks the birth of the electroweak theory , credited to Glashow, Salam and Weinberg $[10,11,12]$. As long as the boson fields are mass less, this would make sense. However, the short range of the massive weak interaction bosons, is orders of magnitude larger than the range of the mass less electromagnetic boson. Without an explanation of this mismatch the pairing of $W_{\mu}^{z}$ and $A_{\mu}$ wouldn't be meaningful. How to give an explanation for the mass of the weak interaction bosons? At this 
point, Weinberg proposed a paradigm shift in particle physics theory [12]. He did so by building out some basic steps made before by various theorists.

The first one is giving a bias to the field of a particle in free space. Its impact becomes apparent in a Lagrangian analysis. Generically, the Lagrangian density L of a bosonic field has the format

$$
L=-\frac{1}{2} \partial_{\mu} \Phi \partial^{\mu} \Phi+U(\Phi)+\rho \Phi
$$

where $U(\Phi)$ is the potential energy of the background field and where $\rho \Phi$ is the source term. A shielded field $\Phi_{D B}$ would have the format,

$$
\Phi_{D B}=\Phi_{0} \frac{\exp \left(-\lambda_{D B} r\right)}{\lambda_{D B} r}
$$

which can be derived by application of the Euler-Lagrange equation from (27) if

$$
U_{D B}=\lambda_{D B}^{2} \frac{\Phi^{2}}{2}
$$

Such a field presupposes a particular energetic background field, such as described by Debije[13]. This field has no bias, because $U_{D B}=0$ for $\Phi=0$. Higgs [14] and independently Englert and Brout [15], hypothesized a bias to the background energy field that results in a typical field format that later has been dubbed as the "Higgs field". This field has the format,

$$
U(\Phi)=-\lambda_{H}^{2} \Phi^{2}+\frac{\mu_{H}^{2}}{2} \Phi^{4}
$$

This broken field is zero for $\Phi_{0}=\left(\lambda_{H} / \mu_{H}\right) \sqrt{2}$.

For the proper understanding of the relationship between the canonical theory and the view from the quark modeling as a third type Dirac particle, it is useful to compare the Debije field (30) with the Higgs field (29). In both cases the field is a background field, which, particularly in the case of (30), should not be confused with the energetic field spread by a massive photon as hypothesized in Proca's generalization of electromagnetism. In the case of Debije's theory, it is the influence of the polarized ionic plasma that shields the mass less boson field from the electric charged pointlike source. Nevertheless, the result is the same as if it were the Proca field of a massive photon. This is a first remark. A second remark has to do with the semantic difference between the spinor space and the momentum space as illustrated in figure 1 . It has been emphasized that the mapping $\left\{p_{0}, p_{1}, p_{2}, p_{3}\right\} \rightarrow\left\{\Psi_{0}, \Psi_{1} \Psi_{2}, \Psi_{3}\right\}$ is not one-to-one. This is due to the twisting of components in consequence of Dirac's model. As stated in chapter 2 , this twist is the origin of the two dipole moments. A $\Phi$ field vector can be regarded as the energetic state of a nuclear particle, such as a quark. This $\Phi$ vector can be regarded as a one-to-one mapping of 
the vectorial momentum sum. Similarly as the angle between two momentum vectors is a measure for its energetic state, an equivalent angle between two field vectors $\Phi_{1}$ and $\Phi_{2}$ may do. The two of these can be captured by a single complex field $\Phi *$, such that

$$
\Phi *=\Phi_{1}+\mathrm{i} \Phi_{2}
$$

Similarly as the angle between the two momentum vectors in momentum space shifts under influence of a boson field $\boldsymbol{A}$ the angle between the field components, $\Phi_{1}$ and $\Phi_{2}$ may shift. As a consequence, the energetic state of the particle will change. The energetic state of the ensemble of the field ensemble is represented by the Lagrangian density as formulated by (27). Applying the same rules of interaction as in the spinor space, the new state of energy can be found under use of the axiom as formulated in (14). All what is needed is defining a covariant derivative that replaces the normal one, followed by insertion into the Lagrangian. Hence, after defining in spinor space,

$$
\partial_{\mu} \rightarrow \partial_{\mu}+g A
$$

and insertion in the Lagrangian composition due to a Debije-type background field, we get,

$$
L=-\frac{1}{2}\left(\partial_{\mu}-\mathrm{i} g A_{\mu}\right) \Phi\left(\partial^{\mu}+\mathrm{i} g A^{\mu}\right) \Phi^{*}-\frac{1}{2} \lambda_{D B}^{2} \Phi \Phi^{*}-\frac{1}{16 \pi} F^{\mu v} F
$$

Elaboration of this expression gives,

$$
L=-\frac{1}{2} \partial_{\mu} \Phi \partial^{\mu} \Phi^{*}+g^{2} A_{\mu} A^{v}-\frac{1}{2} \lambda_{D B}^{2} \Phi \Phi^{*}-\frac{1}{16 \pi} F^{\mu v} F
$$

As compared with the Lagrangian with a zero background field $U(\Phi)=0$, a massive component $g^{2} A_{\mu} A^{v}$ shows up. It seems as if the Debije background field has changed a mass less photon as if it were a boson with mass. The proper interpretation is that the free boson field spread by the nuclear particle is shielded by a background field. As discussed before, the spatial expression of this field, as shown in (28) can be readily obtained from (27). The Debije decay parameter $\lambda_{D B}=\left(m_{D B} c^{2}\right) / \hbar c$ can be interpreted in terms of the energetic quantum of the background field. In this particular case, the origin of this boson can be traced back to a well understood physical process. However, in vacuum electromagnetic fields are not shielded. This might be different for the force interaction fields between nuclear particles. So, let us try putting the origin of the Higgs field and the mass attribute of the weak interaction bosons into this perspective. As discussed before, the meson bond between a quark and an antiquark must be the result of a short range interaction force. The possible equivalent energetic states of this bond justifies the hypothesis of three interaction fields. Similarly as the bosons that carry the electric field spread by an electric charge in an ionic plasma seem to get mass from a background energy (albeit that this mass is just virtual), interaction bosons might get their mass from an energetic background field as well. However, if this field were a symmetric one, there would be no reason why mass less interaction bosons would gain non-virtual mass. For this reason it has been hypothesized that the symmetry break (30) in the field might give an explanation. The proposed symmetry 
break is the change of a symmetrical Debije type background field into the asymmetrical Higgs field. Interaction of mass less bosons with the field around its bias, splits the bosons into two different types. One of the field components remains mass less (as a consequence of Goldstone's theorem $[16,17])$ and the other one gives mass to the electromagnetic boson. By giving a proper bias to the angle in the covariant derivative (20), the mass less boson disappears.

However, giving mass to an electromagnetic boson has not been Weinberg's aim. The electromagnetic boson should remain mass less, while the mass less weak interaction boson in the covariant derivative (26) should gain mass. The approach to obtain this goal marks the second step in Weinberg's approach. To accommodate the weak interaction bosons, a single angle between the field components is not enough and the field needs additional components. Moreover, the covariant derivative has to be extended. In Weinberg's theory to,

$D_{\mu} \Phi=\left\{\partial_{\mu}+\operatorname{ig}^{\prime} \frac{\mathrm{Y}}{2} B_{\mu}+\mathrm{ig}^{\prime \prime}\left(\tau_{1} W_{\mu}^{+}+\tau_{2} W_{\mu}^{-}+\tau_{0} W_{\mu}^{0}\right)\right\} \Phi ; \frac{g^{\prime}}{g^{\prime \prime}}=\tan \vartheta_{W}$

where $g^{\prime 2}+g^{\prime \prime 2}=g^{2}$.

In a particular ratio of the mix, part of the bosons gets mass while another part gets not. The mix is characterized by the matrix,

$\left[\begin{array}{c}\boldsymbol{A}_{\boldsymbol{e m}} \\ \boldsymbol{Z}\end{array}\right]=\left[\begin{array}{cc}\cos \vartheta_{W} & \sin \vartheta_{W} \\ -\sin \vartheta_{W} & \cos \vartheta_{W}\end{array}\right]\left[\begin{array}{c}\boldsymbol{B} \\ \boldsymbol{W}_{\boldsymbol{0}}\end{array}\right]$.

The matrix shows that the present mass less electromagnetic field $\boldsymbol{A}_{\boldsymbol{e} m}$ and the present massive neutral weak interaction field $\boldsymbol{Z}$ are a mix of originally mass less fields $\boldsymbol{B}$ and $\boldsymbol{W}_{\boldsymbol{0}}$. From (36),

$$
\begin{aligned}
& \boldsymbol{A}_{\boldsymbol{e} \boldsymbol{m}}=\boldsymbol{B} \cos \vartheta_{W}+\boldsymbol{W}_{\boldsymbol{0}} \sin \vartheta_{W} \\
& \boldsymbol{Z}=-\boldsymbol{B} \sin \vartheta_{W}+\boldsymbol{W}_{\boldsymbol{0}} \cos \vartheta_{W} .
\end{aligned}
$$

Multiplying the upper equation with $\sin \vartheta_{W}$, the lower one with $\cos \vartheta_{W}$ and addition gives $\boldsymbol{W}_{\boldsymbol{0}}$. Multiplying the upper one $\cos \vartheta_{W}$ etc., gives $\boldsymbol{B}$. Hence,

$$
\boldsymbol{W}_{\boldsymbol{o}}=\boldsymbol{Z} \cos \vartheta_{W}+\boldsymbol{A}_{\boldsymbol{e} \boldsymbol{m}} \sin \vartheta_{W} \text { and } \boldsymbol{B}=-\boldsymbol{Z} \sin \vartheta_{W}+\boldsymbol{A}_{\boldsymbol{e} \boldsymbol{m}} \cos \vartheta_{W}
$$

Note that the matrix (36) only shows the mix condition and that it does not contain mass information. Neither does the covariant derivative. The electromagnetic part of the covariant derivative is partly due to $B_{\mu}$ and partly due to $W_{\mu}^{0}$. From (38) and (35),

$$
g^{\prime} \frac{\mathrm{Y}}{2} B_{\mu}+g^{\prime \prime} \tau_{0} W_{\mu}^{0}=g^{\prime} A_{v}^{e m}\left(\frac{\mathrm{Y}}{2} \sin \vartheta_{W}\right)+g^{\prime} \tau_{0} A_{v}^{e m} \sin \vartheta_{W}+\text { terms without } A_{v}^{e m} .
$$


It means that the electromagnetic coupling factor $e$ is related by the weak interaction coupling factor $g$ by,

$e=g^{\prime} \sin \vartheta_{W}$.

It also means that the electric charge of the quark is given by

$Q=e\left(\frac{\mathrm{Y}}{2}+I_{z}\right) ; \quad I_{z}= \pm \frac{1}{2}$

where $I_{z}$ is the eigen value of the weak isospin component $\tau_{0}$.

It has to be emphasized here that (40) does not apply to $g^{\prime \prime}$, defined in (35). The latter is, in the Standard Model, related with the Fermi constant (10).

Note that the hypercharge $\mathrm{Y}$ just gives a bias to the electric charge of the quark, because the polarity of the charge is determined by $I_{z}$. Including the electron in this theory, imposes for the electron the values $\mathrm{Y}=-1$ and $I_{z}=-1 / 2$. Where an electron shows a single polarity to $I_{z}$, a quark shows two. This can be interpreted from the structural model of figure 1 from the consideration that an electron doesn't have a polarisable dipole moment in a scalar potential field (in Dirac's theory, the electrical dipole moment shows up as an imaginary quantity). This explains why the electron can only assume the weak isospin condition $I_{z}=-1 / 2$. Unlike the electron, the archetype quark has two $I_{z}$ values with opposite polarity. It is common to say that an electron does not show isospin, while the archetype quark does. In the Standard Model, the archetype quark with isospin up and its counterpart with isospin down are regarded as different elementary particles, dubbed as, respectively u quark and d quark.

The magnitude of the mixing angle is empirically found from experimentally established energy values of the weak interaction bosons $\boldsymbol{W}^{ \pm}$( $\hbar \omega_{W} \approx 80.4 \mathrm{MeV}$ ) and the $\boldsymbol{Z}$ boson ( $\hbar \omega_{Z} \approx 91.15 \mathrm{MeV}$ ). Because $\boldsymbol{Z}>>\boldsymbol{A}_{\text {em }}$, we have from (38),

$\cos \vartheta_{W}=\frac{\hbar \omega_{W}}{\hbar \omega_{Z}}$

It is worthwhile to note that neither the magnitude of the mixing angle $\vartheta_{W}$ nor the value of the hypercharge $\mathrm{Y}$ is fixed by theory. Later in this article it will be shown that the value of the mixing angle is, in fact, fixed by theory as a consequence of the third type Dirac quark. In the Standard Model, the hypercharge bias has got a value $1 / 3$, for reasons of classification. It is instructive to discuss the hypercharge issue with the structure as shown in figure 1 in mind.

By proper normalization of the potential field $\boldsymbol{A}_{\boldsymbol{e}}$, the coupling factor $g$ can, as a dimensionless quantity, related to $e$ by the fine structure expression, 
$e^{2}=4 \pi \varepsilon_{0} g^{2} \hbar c$

Hence, from (40) and (43)

$e=g^{\prime} \sin \vartheta_{W}=g \sqrt{4 \pi \varepsilon_{0} \hbar c} \rightarrow \frac{g^{\prime}}{g}=\frac{\sqrt{4 \pi \varepsilon_{0} \hbar c}}{\sin \vartheta_{W}}$

Note that $\boldsymbol{A}_{e m}$ and $\boldsymbol{Z}$ both are four-dimensional fields. Curiously, while the $A_{0}^{e m}$ component of the $\boldsymbol{A}_{\boldsymbol{e}}$ field is easily recognized as a potential field, the $Z_{0}$ component of the $\boldsymbol{Z}$ field is usually not considered. Hence, the awareness of a nuclear potential field is lacking in the Standard Model. In the structural model shown in figure 1, it is the monopole field of a quark such as expressed by (8). Other quarks may couple to this field, similarly as electrons couple to the field of other electrons. Also here, I prefer using a dimensionless coupling constant to such a field.

To establish the nuclear equivalent of the electromagnetic potential, the far field force $F_{F}$ evoked by a quark is compared with the electromagnetic force $F_{e}$. Generally, under consideration of (8),

$F_{e}=-e \frac{\partial}{\partial r} \frac{e}{4 \pi \varepsilon_{0} r} \quad$ and $\quad F_{F}=-g \frac{\partial}{\partial r} \Phi_{0} \frac{\exp (-\lambda r)}{\lambda r}$

There is no reason why these forces would be the same. What is clear, however, is, that $g \Phi_{0} / \lambda$ plays a similar role as $e^{2} /\left(4 \pi \varepsilon_{0}\right)$, i.e.,

$\frac{e^{2}}{4 \pi \varepsilon_{0}} \leftrightarrow \frac{g \Phi_{0}}{\lambda}$

Using a dimensionless coupling constant $g$, as defined by (43) is a suitable and allowable choice, because any other choice can be accommodated in the quantities $\Phi_{0}$ and $\lambda$. The dimensionality of $g$ is compatible with the dimension $\Phi_{0}$ in units of energy and with the $\mathrm{m}^{-1}$ dimensionality of $\lambda$.

The hypercharge

Apart from the strong interaction dipoles, the figure shows the common nuclear dipole set. While the orientation of the strong interaction dipole is fixed by polarization, the nuclear dipole caused by the elementary angular momentum is still subject to spin statistics. Table II shows the possible nuclear spin configurations. In the convention that I wish to adopt within the scope of this article, a quark in clockwise spin will be regarded as a quark in up condition, to be coded as $u$, and a quark in anticlockwise spin will be coded as $d$. This is different from the coding convention in the Standard Model where $u$ and $d$ quarks are regarded as different particles. For distinction, for the convention in this article italics $u$ and $d$ are used, and regular $u$ and $d$ for the Standard Model convention. 
The spin states of a two quantum mechanical particles in conjunction establish a triplet state $|1, \pm 1\rangle$ or $|1,0\rangle$ next to a singlet state $|0,0\rangle$. Where the pseudoscalar state of the quark junction shows the triplet state only, the vector state shows the singlet next to the triplet. Due to the third-type Dirac properties, the quark has two dipole moments that are both subject to spin statistics. In spite of the polarization constraint, the polarisable dipole moment is still subject to these spin statistics. This spin will be denoted as isospin. From figure 1 it is obvious that the polarisable dipoles (isospins) of the particle are oriented in the same direction. The sign of the direction may change, even to the extent that the unidirectional sign may be mixed up. This is reflected in the third column of Table II. The table shows the possible nuclear spin states and isospin states of the archetype meson. It also shows how the electric charge of the meson can be found from the isospin condition. The coding is based upon the status of isospin. The table suggests by hypothesis that this status of the polarisable dipole, hence isospin, is the true cause of electric charge. Hence, the electric charge might be a holistic attribute of the meson, rather than a sum of individual contributions. This is different from the Standard Model, where a bias $\mathrm{Y}=1 / 3$ makes two different quarks, each with their own charge. Table III shows the composition of the archetype meson as conceived in the Standard Model.

Table II

\begin{tabular}{|c|c|l|l|l|l|l|l|c|}
\hline meson & $q_{u}$ & $\bar{q}_{u}$ & isospin & bias & & $\begin{array}{l}\text { pseudo } \\
\text { scalar }\end{array}$ & vector & $\mathrm{Q}$ \\
\hline \multirow{5}{*}{$q_{u} \bar{q}_{u}$} & $\uparrow$ & $\downarrow$ & $\uparrow \uparrow$ & 0 & $u \bar{d}$ & $\pi^{+}$ & & 1 \\
\cline { 2 - 9 } & $\downarrow$ & $\uparrow$ & $\downarrow \downarrow$ & 0 & $d \bar{u}$ & $\pi^{-}$ & & -1 \\
\cline { 2 - 9 } & $\uparrow \downarrow$ & $\downarrow \uparrow$ & $\uparrow \uparrow+\downarrow \downarrow$ & 0 & $u \bar{u}+d \bar{d}$ & $\pi^{0}$ & & 0 \\
\cline { 2 - 9 } & $\uparrow$ & $\uparrow$ & $\uparrow \uparrow$ & 0 & $u \bar{u}$ & & $\rho^{+}$ & 1 \\
\cline { 2 - 9 } & $\downarrow$ & $\downarrow$ & $\downarrow \downarrow$ & 0 & $d \bar{d}$ & & $\rho^{-}$ & -1 \\
\cline { 2 - 9 } & $\uparrow \downarrow$ & $\uparrow \downarrow$ & $\uparrow \uparrow+\downarrow \downarrow$ & 0 & $u \bar{u}+d \bar{d}$ & & $\rho^{0}$ & 0 \\
\cline { 2 - 9 } & $\uparrow \downarrow$ & $\downarrow \uparrow$ & $\uparrow \uparrow+\downarrow \downarrow$ & 0 & $u \bar{u}+d \bar{d}$ & & $\omega$ & 0 \\
\hline
\end{tabular}

Table III

\begin{tabular}{|l|l|l|l|l|l|l|l|}
\hline meson & $q_{u}$ & $\bar{q}_{u}$ & bias & & $\begin{array}{l}\text { pseudo } \\
\text { scalar }\end{array}$ & vector & $\mathrm{Q}$ \\
\hline \multirow{4}{*}{$q_{u} \bar{q}_{u}$} & $\mathrm{u}$ & $\overline{\mathrm{d}}$ & $1 / 3$ & $\mathrm{u} \overline{\mathrm{d}}$ & $\pi^{+}$ & $\rho^{+}$ & 1 \\
\cline { 2 - 8 } & $\mathrm{d}$ & $\overline{\mathrm{u}}$ & $1 / 3$ & $\mathrm{~d} \overline{\mathrm{u}}$ & $\pi^{-}$ & $\rho^{-}$ & -1 \\
\cline { 2 - 8 } & $\mathrm{u} / \overline{\mathrm{d}}$ & $\mathrm{d} / \overline{\mathrm{u}}$ & $1 / 3$ & $\mathrm{u} \overline{\mathrm{d}}-\mathrm{d} \overline{\mathrm{u}}$ & $\pi^{0}$ & $\rho^{0}$ & 0 \\
\hline
\end{tabular}

The difference between the two tables is due to the assignment of the hypercharge. As noted above, the electroweak theory is not decisive on this issue. In the Standard Model, the hypercharge has been heuristically established from the hadron classification model, as proposed in 1964, independently by Gell-Mann [6] and Zweig [18]. The bias allowed a 
streamlining of the charges of mesons with those of baryons. However, at the time only $u, d$ and s quarks were known. After the discovery of more flavors, the charge formula in table I has been heuristicly adapted by including more quantum numbers. In chapter 4 the issue will be rediscussed, including the observation that, while the archetype quark shows two different states of isospin, the non-archetypes only show a single state.

\section{Spatial expression of the Higgs field}

Similarly as in the case of the Debije field example, it would be nice to find a spatial expression for the Higgs field. In principle, it can be done by means of the Lagrange-Euler equation, by supposing that a quark is an energetic pointlike source. Because of the high dominance of the weak interaction over the electromagnetic interaction, the influence of the latter can be first ignored and later be added as a second order effect. Hence, the scalar part of the weak interaction potential determines the stationary condition of the spatial field. Unfortunately the high non-linearity of the Higgs field prevents deriving an analytical solution $\Phi(r)$ from (27) and (30). However, a numerical procedure allows deriving a twoparameter expression for $\Phi(r)$ that closely approximates a true analytical solution. In this approach a generic Ansatz format is adopted for $\Phi(r)$ from which an expression is retrieved of $U(\Phi)$. Subsequently, a fit of is searched on (30). In this approach, first of all, the EulerLagrange equation is applied on the static Lagrangian density (27). Hence, from

$$
\frac{\partial \mathrm{L}}{\partial \Phi}-\partial_{i}\left(\frac{\partial \mathrm{L}}{\partial\left(\partial_{i} \Phi\right)}\right)=0
$$

we have from (27),

$$
\partial_{i} \Phi \partial^{i} \Phi=\frac{d}{d \Phi} U(\Phi)+\rho
$$

The Ansatz format of the field $\Phi(r)$ is chosen as,

$$
\Phi(r)=\Phi_{0} \frac{\exp (-\lambda r)}{\lambda r}\left\{\frac{\exp (-\lambda r)}{\lambda r}-1\right\}
$$

Substitution of (49) into (46) and subsequent calculation of $U(\Phi)$ gives a fit with (30) for $\mu_{H}^{2}$ and $\lambda_{H}^{2}$, such that

$$
\frac{1}{2} \mu_{H}^{2}=1.06 \lambda^{2} \text { and } \frac{1}{4} \lambda_{H}^{2}=32.3 \frac{\lambda^{2}}{\Phi_{0}^{2}}
$$

The rationale behind this choice is the assumption that the inter-quark potential will behave similarly as the inter-nucleon potential [18].

The two-parameter field is indistinguishable from the three-parameter field, 
$\Phi(r)=\Phi_{0} \exp (-\lambda r)\left\{\frac{1}{(\lambda r)^{2}}-w \frac{1}{\lambda r}\right\}$ for $w=1 / 0.555$

The quark's field would show the characteristics as shown in Figure 3. It would imply that a quark would be repelled by any other quark under influence of the far field, but attracted by the near field, thereby giving rise to mesons as stable two-quark junctions and baryons as three-quark junctions.
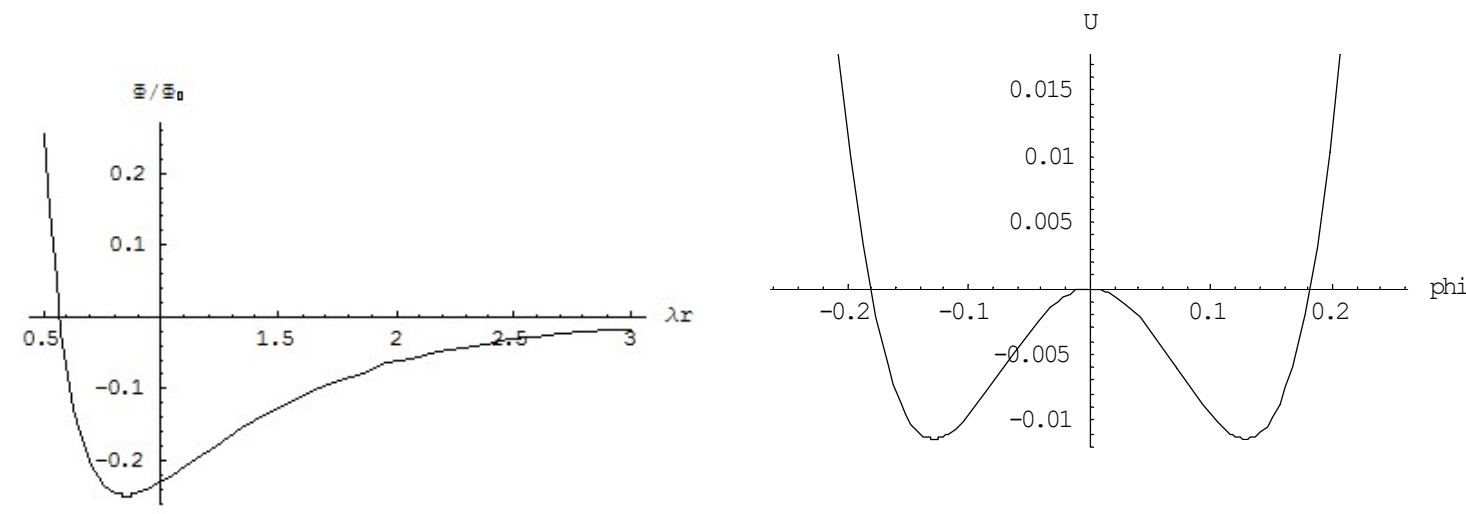

Figure. 3. (Left) The quark's scalar field $\Phi / \Phi_{0}$ as a function of the normalized radius $\lambda x$; (Right) The background field $U_{H}(\Phi)=-U(\Phi)$ retrieved from the spatial expression.

Unfortunately, this radial symmetric format is not viable, because it violates the renormalization constraint. However, comparing (51) with (8) reveals a striking correspondence. Nevertheless, there is a major difference as well. Where the derivation of (50) has been based upon a presupposed energetic monopole model for the quark, (8) is the result of a dipole moment next to a monopole. Hence, by restricting the validity of (51) to the dipole axis $x$, the renormalization problem is removed by rewriting (51) as a sum of a far field and a near field, such that,

$\Phi(x)=\Phi_{F}(x)+\Phi_{N}(x)$ with $\Phi_{F}(x)=-w \Phi_{0} \frac{\exp (-\lambda x)}{\lambda x}$ and $\Phi_{N}(x)=\Phi_{0} \frac{\exp (-\lambda x)}{(\lambda x)^{2}}$

The conclusion therefore is that the Higgs field has to be interpreted as the shielded radial symmetric field of an energetic monopole in conjunction with a one-dimensional dipole field. The quark, conceived as a third-type Dirac particle is compatible with this model. The near field is due to the dipole and gives an interpretation for the near field that, although implicitly present in the electroweak theory, is usually not recognized as the strong interaction glue that binds the quarks together in hadronic structures. The break in the field that spoils the symmetry of the Debije field, thereby modifying it into the Higgs field, can be ascribed to the quark's polarisable dipole moment. One may speculate if a quark has already shown the similar characteristic at the early time of the break-up of the universal energy into electromagnetic energy and nuclear energy. 


\section{The meson model}

Let us proceed by describing the bond between a quark and an antiquark on the basis of the potential field of a quark that has been derived from the modeling of the quark as a third type Dirac particle, which resulted into the field expression, shown by (51). Conceiving the pion as a structure in which a quark couples to the field of the antiquark with the generic quantum mechanical coupling factor $g$, the pion can be modeled as the one-body equivalent of a two-body oscillator, described by the equation for its wave function $\psi$,

$-\frac{\hbar^{2}}{2 m_{m}} \frac{\mathrm{d}^{2} \psi}{\mathrm{dx}^{2}}+\{U(d+x)+U(d-x)\} \psi=E \psi ; \quad U(x)=g \Phi(x)$

where $\hbar$ is Planck's reduced constant, $2 d$ the quark spacing, $m_{m}$ is the bare mass of the quark (not to be confused with the constituent mass), $V(x)=U(d+x)+U(d-x)$ its potential energy, and $E$ the generic energy constant, which is subject to quantization. It will be clear from (49) that the potential energy $V(x)$ can be expanded as,

$$
V(x)=U(d+x)+U(d-x)=g \Phi_{0}\left(k_{0}+k_{2} \lambda^{2} x^{2}+\ldots .\right),
$$

where $k_{0}$ and $k_{2}$ are dimensionless coefficients that depend on the spacing $2 d$ between the quarks.

In spite of the resemblance with a classical quantum mechanical oscillator, the model is relativistic, because the mass in the wave equation does not represent the individual masses of the two bodies. Instead, it is an equivalent mass that captures the energy of the field. Furthermore, it has to be kept in mind that this model holds in the center of mass frame, so that a lab frame interpretation will need a relativistic correction. To facilitate the analysis, (53) is normalized as,

$-\alpha_{0} \frac{\mathrm{d}^{2} \psi}{\mathrm{d} x^{\prime 2}}+V^{\prime}\left(x^{\prime}\right)=E^{\prime} \psi$

where $\alpha_{0}=\frac{\lambda^{2} \hbar^{2}}{2 m_{m} g \Phi_{0}}, x^{\prime}=x \lambda, d^{\prime}=d \lambda, E^{\prime}=\frac{E}{g \Phi_{0}}, U^{\prime}\left(x^{\prime}\right)=\frac{U(\lambda x)}{g \Phi_{0}}$ and

$V^{\prime}\left(x^{\prime}\right)=U^{\prime}\left(d^{\prime}+x^{\prime}\right)+U^{\prime}\left(d^{\prime}-x^{\prime}\right)=k_{0}+k_{2} x^{\prime 2}+\ldots \ldots$

Moreover, from [20, eq. (C2),9],

$\alpha_{0}=\frac{k_{0}^{2}}{2 k_{2}}$

Normalized quantities in this text will be indicated by a "prime" (').The coefficients $k_{0}\left(d^{\prime}\right)$ and $k_{2}\left(d^{\prime}\right)$ can be straightforwardly calculated from (56) and (49) as, 


$$
\begin{aligned}
& k_{0}=2\left(\frac{\exp \left(-2 d^{\prime}\right)}{d^{\prime 2}}-\frac{\exp \left(-d^{\prime}\right)}{d^{\prime}}\right) \\
& k_{2}=\frac{\exp \left(-2 d^{\prime}\right)}{d^{\prime 4}}\left(6+4 d^{\prime 2}+8 d^{\prime}\right)-\frac{\exp \left(-d^{\prime}\right)}{d^{\prime 2}}\left(2+d^{\prime}+\frac{2}{d^{\prime}}\right) .
\end{aligned}
$$

The two quarks in the meson settle in a state of minimum energy, at a spacing $2 \lambda d=2 d_{\text {min }}^{\prime}$, such that $[20,21]$,

$$
d_{\min }^{\prime}=\lambda d=0.853 ; k_{0}=-1 / 2 \text { and } k_{2}=2.36
$$

Note: the field format (49) has been preferred above the indistinguishable field format (52) because (49) is a two-parameter format, while (52) is a three-parameter one.

The archetype, the pion, is the two-quark oscillator in its ground state. The first excitation state transforms a pion into a kaon. The mass ratio between the two is the same as the mass ratio of the normalized energy constants $E^{\prime}-k_{0}$. This is not trivial and it reflects the basic theorem of the theory. This theorem states that the energy wells of the two quarks are not massive. Instead, the mass attribute of two-quark junctions (mesons) and three-quark junctions (baryons) is made up by the vibration energy as expressed by the energy state of the quantum mechanical oscillator that they build. The distribution of this mass over constituent quarks is a consequence of this mechanism. Unfortunately, an analytical calculation of the $E^{\prime}-k_{0}$ ratio of kaons over pions, is only possible for the quadratic approximation of the series expansion of the potential energy $V^{\prime}\left(z^{\prime}\right)$. A more accurate calculation requires a numerical approach. A procedure to do so has been documented in [20, Appendix C]. It shows that some simple lines of code in Wolfram's Mathematica [22] may do the job. The numerically calculated ratio of the energy constants appears to be 3.57 instead of 3 as it would have been in the harmonic case. The result explains the excitation of the $137 \mathrm{MeV} / \mathrm{c}^{2}$ pion mass to the $490 \mathrm{MeV} / \mathrm{c}^{2}$ mass of the pseudoscalar kaon. This result gives a substantial support for the viability of the theory as will be further developed in this article. This result also gives rise to the question if other mesons can be regarded as a result from enhanced excitation. Table IV gives a survey of the calculated ratios for higher excitation ratios. It gives the pseudoscalar $\eta^{\prime}$ meson as a candidate from second level excitation. The table gives no candidate for third level excitation. As shown in [23], the corresponding level of energy would imply a meson state with a positive value for the binding energy (as is reflected in the value of $k_{0}$ ), which prevents a sustainable quasi-stable configuration.

In this calculation, the electromagnetic interactions have been ignored, because their influence is considered to be of second order as compared to the nuclear interaction. More on this will be subject of chapter 9 . Interestingly, the kaon energy does not only correspond with the energy of a pion in its state of first excitation, but also with the ground state energy of a quark junction at smaller spacing, thereby composing the kaon as a $u \bar{s}$ or a $\bar{u} s$ bond, composed by the $u$ quark next to the heavier $s$ quark, such as illustrated in figure Therefore, the excitation mechanism is potentially subject to bootstrapping. It shows many excitation and de-excitation routes, resulting in a quasi-chaotic, but nevertheless deterministic, mass spectrum of mesons. 
Table IV: meson excitations

\begin{tabular}{|l|l|l|l|}
\hline Bottom level & $E_{\text {bind }}^{\prime}=-1 / 2$ & mass ratio & mass in $\mathrm{MeV} / \mathrm{c}^{2}$ \\
\hline Ground state & $E_{0}^{\prime}-E_{\text {bind }}^{\prime}=0.84$ & 1 & $\begin{array}{l}137 \\
\text { (pion = 135-140) }\end{array}$ \\
\hline First excitation & $E_{1}^{\prime}-E_{\text {bind }}^{\prime}=3.00$ & 3.57 & $\begin{array}{l}489 \\
(\text { kaon }=494-498)\end{array}$ \\
\hline Second excitation & $E_{2}^{\prime}-E_{\text {bind }}^{\prime}=6.06$ & 7.21 & $\begin{array}{l}988 \\
\left(\eta^{\prime}=958\right)\end{array}$ \\
\hline Third excitation & $E_{3}^{\prime}-E_{\text {bind }}^{\prime}=9.94$ & 11.83 & $? ? ?$ \\
\hline
\end{tabular}

So, the meson's mass spectrum can be explained from excitations of a basic structure consisting of two identical quarks. The pion-kaon-etaprime sequence is just the hat-stand of a framework for the assignment of constituent quark masses. Next to this excitation mechanism, there is another major influence on the mass of mesons. This is interaction of nuclear spins between the two quarks. This mechanism will be discussed in the next chapter. It causes a major difference between the masses of pseudoscalar mesons and vector mesons. The result of this influence is shown in figure 5 .

This is beyond the scope in this article. Details on this and more about mass excitation can be found in [23], which is an update of [20].

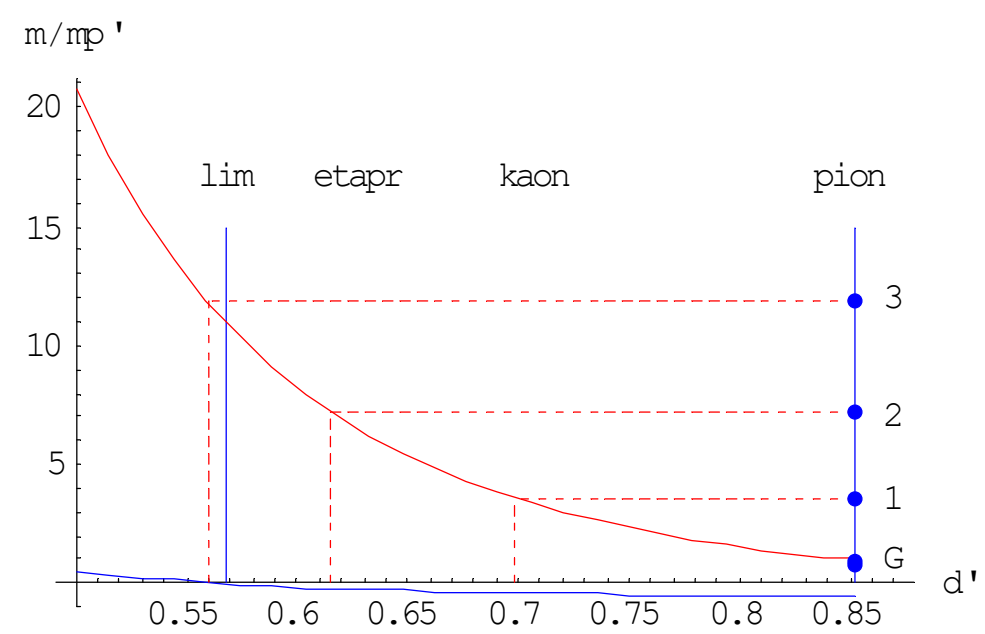

Figure 4. The light sector limit. The graph shows the increase of the massive energy of a quark/antiquark pair relative to the pion state as a function of the quark spacing. Two excitation levels beyond the pion's ground state are converted into the ground state of, respectively, the kaon and the $\eta^{\prime}$, thereby producing the $(u, s)$ - quark family. Third level excitation is prevented by the loss of binding energy (lower curve).

Table $\mathrm{V}$ shows the meson classification based upon isospin coding as used before in Table II . Note the difference between the italic coding as used in this article and the regular coding as used in the $P$ (article)D(ata)G(roup) tables. The upper part of Table $V$ is just another representation of the "octet" classification by Gell-Mann and Murray (the Eightfold Way). 


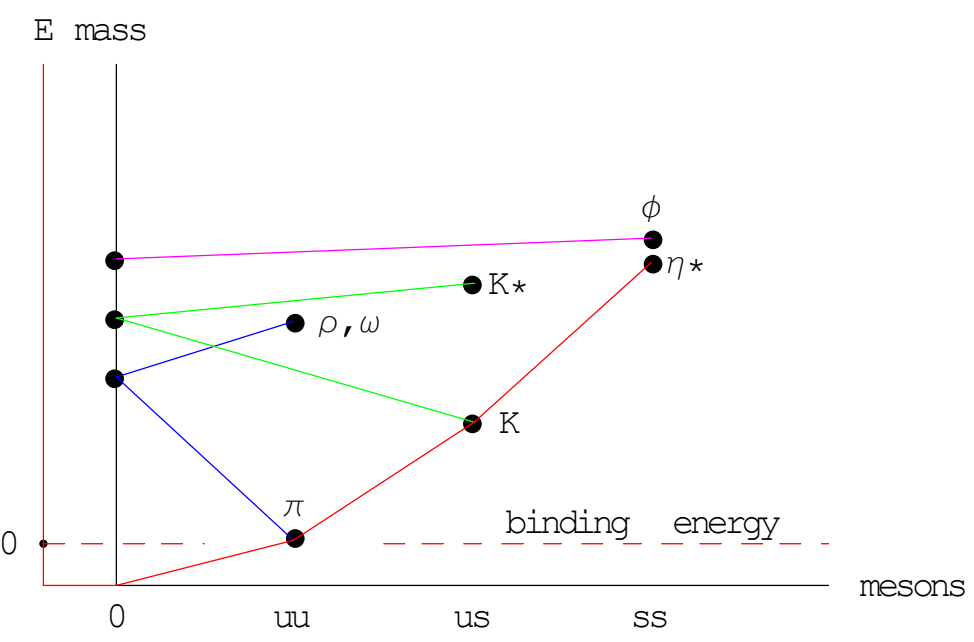

Figure5: Illustration of the influence of the nuclear spin interactions between the quarks in the light sector. The black dots on the vertical axis represent the sum of the constituent masses in, respectively, the ud, us and ss mesons.

Table V: meson classification

\begin{tabular}{|c|c|c|c|c|c|c|}
\hline meson & $\begin{array}{l}\text { Isospin } \\
\text { modes }\end{array}$ & & recode & $Q$ & $\begin{array}{l}\text { pseudo } \\
\text { scalar }\end{array}$ & vector \\
\hline \multirow{4}{*}{$u \bar{u}$} & $\uparrow \uparrow$ & $u \bar{u}$ & $\mathrm{ud}$ & 1 & $\pi^{+}$ & $\rho^{+}$ \\
\hline & $\downarrow \downarrow$ & $d \bar{d}$ & $\overline{\mathrm{u} d}$ & -1 & $\pi^{-}$ & $\rho^{-}$ \\
\hline & \multirow{2}{*}{$\uparrow \downarrow \pm \downarrow \uparrow$} & $(u \bar{u}-\bar{d} d) / \sqrt{2}$ & $(u \bar{u}-\bar{d} d) / \sqrt{2}$ & 0 & $\pi^{0}$ & $\rho^{0}$ \\
\hline & & $(u \bar{u}+\bar{d} d) / \sqrt{2}$ & $(u \bar{u}+\bar{d} d) / \sqrt{2}$ & 0 & $x$ & $\omega$ \\
\hline \multirow[t]{2}{*}{$u \bar{s}$} & $\uparrow \uparrow$ & $u \bar{s}$ & $\mathrm{u} \overline{\mathrm{s}}$ & 1 & $\mathrm{~K}^{+}$ & $\mathrm{K}^{*+}$ \\
\hline & $\downarrow \uparrow$ & $d \bar{s}$ & $\mathrm{~d} \overline{\mathrm{s}}$ & 0 & $\mathrm{~K}^{0}$ & $\mathrm{~K}^{* 0}$ \\
\hline \multirow[t]{2}{*}{$\bar{u} s$} & $\uparrow \downarrow$ & $\bar{u} s$ & $\overline{\mathrm{d} s}$ & 0 & $\overline{\mathrm{K}}^{0}$ & $\overline{\mathrm{K}}^{* 0}$ \\
\hline & $\downarrow \downarrow$ & $\bar{d} s$ & $\overline{\mathrm{us}}$ & -1 & $\overline{\mathrm{K}}^{-}$ & $\overline{\mathrm{K}}^{*-}$ \\
\hline$s \bar{s}$ & $\downarrow \uparrow$ & $s \bar{s}$ & $s \bar{s}$ & 0 & $x$ & $\varphi$ \\
\hline \multirow[t]{2}{*}{$\bar{u} c$} & 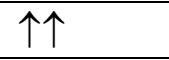 & $\bar{u} c$ & $\overline{\mathrm{d}} \mathrm{c}$ & 1 & $\mathrm{D}^{+}$ & $\mathrm{D}^{*+}$ \\
\hline & $\downarrow \uparrow$ & $\bar{d} c$ & $\overline{\mathrm{u}} \mathrm{c}$ & 0 & $\mathrm{D}^{0}$ & $\mathrm{D}^{* 0}$ \\
\hline \multirow[t]{2}{*}{$u \bar{c}$} & $\uparrow \downarrow$ & $u \bar{c}$ & $\mathrm{u} \overline{\mathrm{c}}$ & 0 & $\mathrm{D}^{0}$ & $\mathrm{D}^{* 0}$ \\
\hline & $\downarrow \downarrow$ & $d \bar{c}$ & $\mathrm{~d} \overline{\mathrm{c}}$ & -1 & $\mathrm{D}^{-}$ & $\mathrm{D}^{*-}$ \\
\hline$s \bar{c}$ & $\downarrow \downarrow$ & $s \bar{c}$ & $s \overline{\mathrm{c}}$ & -1 & $\mathrm{D}_{\mathrm{s}}^{-}$ & $\mathrm{D}_{\mathrm{s}}^{*-}$ \\
\hline $\bar{s} c$ & $\uparrow \uparrow$ & $\bar{s} c$ & $\overline{\mathrm{sc}}$ & 1 & $\mathrm{D}_{\mathrm{s}}^{+}$ & $\mathrm{D}_{\mathrm{s}}^{*+}$ \\
\hline$c \bar{c}$ & $\uparrow \downarrow$ & $c \bar{c}$ & $\mathrm{c} \overline{\mathrm{c}}$ & 0 & $x$ & $\mathrm{~J} / \psi$ \\
\hline
\end{tabular}

The table shows a particular issue of attention. Where the $u / d$ quark shows both polarities of the polarisable dipole moment, read isospin, the scaled quarks violate parity in this respect. These show just a single state of polarity. The origin of this parity difference is not quite clear. It might have to do with the origin of the scaled quarks from the excitation mechanism. In the Standard Model, the problem is heuristically settled by axiom. To illustrate this, it has to be taken into account that a Dirac particle possess a wave function 
that can be split into two parts that are chirally different (Weyl's decomposition of Dirac's wave function [3]). One part is left-handed, the other part is right-handed. The chirality of a particle is opposite to that of an antiparticle. This property has been invoked in this text before by showing that all mesons belong to an SU(2) group. To explain the classification phenomenon just mentioned, it is hypothesized in the Standard Model by axiom that quarks are single-handed, such that each left-handed quark has a right-handed counterpart, just as if they were antiparticles. The u-quark forms a chiral pair with a d-quark, the s-quark forms a pair with the c-quark and the b-quark forms a pair with the t-quark. This suggests that the parity problem is solved by theory. It is true that this solves the classification phenomenon. From a physical point of view, it is not different from the observation that scaled quarks violate the isospin parity.

In [20] it has been documented in detail how the rest masses of the pseudoscalar as well as the vector mesons can be calculated to a rather high precision. The results of the calculation are shown in the lower part of Table VI. The second column shows the constituent mass values of the quarks. Mass values before the / are for the pseudoscalar mesons, behind the / for the vector mesons. The third column shows mesons with a homogeneous composition, the other columns are those with a inhomogeneous composition. Note that the coding is in italic convention as used in this text, in which a $u$ quark is equivalent to a $d$ quark.

Table VI: Calculated meson masses compared with experimental evidence

\begin{tabular}{|l|l|l|l|l|l|}
\hline \multicolumn{5}{|c|}{ experimental evidence } & \\
\hline$m_{u}^{\prime}$ & & $u \bar{u}(775 / 139)$ & $u \bar{u} \pm \bar{u} u(782 /$ n.a. $)$ & & \\
\hline$m_{s}^{\prime}$ & & $s \bar{s}(1020 /$ n.a.) & $s \bar{u}(892 / 489)$ & & \\
\hline$m_{c}^{\prime}$ & & $c \bar{c}(3096 / 2983)$ & $c \bar{s}(2112 / 1968)$ & $c \bar{u}(2007 / 1869)$ & \\
\hline$m_{b}^{\prime}$ & & $b \bar{b}(9460 /$ n.a. $)$ & $b \bar{c}(? / 6276)$ & $b \bar{s}(5415 / 5367)$ & $b \bar{u}(5325 / 5279)$ \\
\hline
\end{tabular}

\begin{tabular}{|l|l|l|l|l|l|}
\hline \multicolumn{7}{|c|}{ calculated } \\
\hline$m_{u}^{\prime}$ & 310 & $u \bar{u}(780 / 140)$ & $u \bar{u} \pm \bar{u} u(780 /$ n.a. $)$ & & \\
\hline$m_{s}^{\prime}$ & 483 & $s \bar{s}(1032 /$ n.a. $)$ & $s \bar{u}(896 / 485)$ & & \\
\hline$m_{c}^{\prime}$ & 1515 & $c \bar{c}(3096 / 2983)$ & $c \bar{s}(2119 / 1988)$ & $c \bar{u}(1996 / 1865)$ & \\
\hline$m_{b}^{\prime}$ & 4720 & $b \bar{b}(9469 /$ n.a. $)$ & $b \bar{c}(6263 / 6263)$ & $b \bar{s}(5400 / 5358)$ & $b \bar{u}(5332 / 5289)$ \\
\hline
\end{tabular}

\section{Bare mass and constituent mass}

Let us now proceed by modelling the quark somewhat further. Because it is a Dirac particle of the third kind, it possesses a second dipole moment with the value $m_{p} d=\hbar / 2 c$, where $m_{p}$ and $d$ are unknown quantities. The individual values are just auxiliary. It has to be emphasized, though, that $m_{p} \neq m_{q u}$,i.e., different from the quark's rest mass $m_{q u}$. This dipole creates along the dipole axis $x$ a gravitational near field potential field $\Phi_{G N}(x)$, such that, 
$\Phi_{G N}(x)=\frac{G m_{p} d}{x^{2}} \rightarrow \Phi_{G N}(x)=\frac{\hbar}{2 c} \frac{\lambda^{2} G}{(\lambda x)^{2}} \rightarrow \Phi_{G N}(x)=\Phi_{0} \frac{1}{(\lambda x)^{2}} ; \Phi_{0}=\frac{\hbar}{2 c} G \lambda^{2}$

where $G$ is the common gravitational constant. Note that $\Phi_{0}$ is energy per unit of mass. Apart from the near field there must be a far field as a result from the mass associated with the elementary momentum $\hbar$. Let us interpret the elementary angular moment $\hbar$ as a spin with eigen value $1 / 2$ as a consequence of a virtual rotation at light speed of the mass $m_{p}$ at a fictitious radius $r_{0}=1 / k \lambda$. Hence,

$\frac{\hbar}{2}=\frac{m_{p} c}{k \lambda} \rightarrow m_{p}=k \frac{\hbar}{2} \frac{\lambda}{c}$

The quantity $k$ is an unknown adaption constant, introduced here to couple the $\lambda$ parameter to the one used in the preceding chapters. Hence, from classical field theory,

$\Phi_{G F}(r)=\frac{m_{p} G}{r}=k \frac{\hbar}{2} \frac{\lambda}{c} \frac{G}{r}=k \frac{\hbar}{2} \frac{G \lambda^{2}}{c} \frac{1}{\lambda r}$

and, under consideration of $\Phi_{0}$ as defined in (59),

$\Phi_{G F}(r)=k \frac{\hbar}{2} \frac{G \lambda^{2}}{c} \frac{1}{\lambda r}=\Phi_{0} \frac{k}{(\lambda r)}$

Hence, if the potential field of the quark modeled as a third type Dirac particle can be expressed as,

$\Phi(\lambda x)=\Phi_{0}\left\{\frac{1}{(\lambda x)^{2}}-k \frac{1}{\lambda x}\right\} ; \quad \Phi_{0}=\frac{\hbar}{2 c} G \lambda^{2}$

According to the theory developed in the previous chapters, a quark feels a force from another quark as,

$F=g \frac{\partial}{\partial r} \Phi_{\text {qu }}$

The format of the field $\Phi_{q u}$ matches with the format of the field (63). Because $k$ is a degree of freedom, we may equate $\Phi_{q u}=\Phi(\lambda x)$.

Hence, expressing the gravitational force between the quarks as an equivalent nuclear force,

$F=g \frac{\partial}{\partial r} \Phi=m_{q u} \frac{\partial}{\partial r} \Phi_{\mathrm{G}}$ 
From (61) and considering that $\Phi_{0}$ in (64) is energy per unit of mass, we get from (65),

$\rightarrow g\left(k m_{q u} \frac{\hbar}{2} \frac{G \lambda^{2}}{c}\right)=m_{q u}^{2} k G \lambda \rightarrow m_{q u}=g \frac{\hbar}{2 c} \lambda$.

This mass quantity determines the strength of the gravitational interaction in relation to the weak interaction strength. The quark's bare mass can be found from the relativistic correction of this quantity from the center of mass frame to the lab frame. This can be done by establishing the relationship between the $\lambda$ value in the center of frame with the $\lambda$ value in the lab frame. This is possible by considering that the spacing between the quarks in the center of frame is equal or near to the half wavelength of a boson $\hbar \omega_{W}$. Considered, however, in the inertial frame, the energy of the boson is the rest mass of the meson $\hbar \omega_{\pi}$. Hence

$2 d_{\min }=\frac{1}{2} \alpha c T=\frac{1}{2} \alpha \hbar c \frac{2 \pi}{\hbar \omega_{W}}=2 \frac{d_{\min }^{\prime}}{\lambda} \rightarrow \lambda_{c o m}=\frac{1}{\pi} \frac{\hbar \omega_{W}}{\hbar c} 2 d_{\min }^{\prime} \rightarrow \lambda=\frac{2\left(\hbar \omega_{\pi}\right) d_{\min }^{\prime}}{\alpha \pi(\hbar c)}$.

The dimensionless factor $\alpha$ is of order 1 . Its numerical value $\alpha \approx 0.69$ has been assessed from an application of this meson theory that enabled to calculate the gravitational constant $G$ in terms of quantum mechanical quantities [21].

From (66) and (67) the quark's bare massive energy amounts to,

$$
m_{q u} c^{2}=c^{2} g \frac{\hbar}{2 c} \frac{1}{\alpha \pi} \frac{\hbar \omega_{\pi}}{\hbar c} 2 d_{\min }^{\prime}=\frac{g d_{\min }^{\prime}}{\alpha \pi} \hbar \omega_{\pi}(\approx 2.6 \mathrm{MeV})
$$

This is in agreement with the values reported by PDG (Particle Data Group).

Where the bare mass is evoked by the elementary dipole moment $\hbar / c$, the massive energy of the pion's center of mass is due to the quantum mechanical oscillator state that results from the equilibrium of forces made up by the nuclear field spread by the quarks. This oscillator is, in fact, a one-body equivalent of a two-body one. Hence the massive energy of the center of mass can be interpreted in terms of constituent masses of the two quarks. Where we have considered so far the role of the polarisable dipoles of those masses that, like we have seen, create the strong interaction force, there still is a first dipole moment as well, which can be interpreted as the nuclear equivalent of the magnetic moment of an electron. Similarly as the magnetic moment of the electron in its orbit around a proton or positron interacts with the magnetic moment of the proton or positron, the nuclear moments of the quark interact. For its analysis, I'll assume Griffith's model as a starting point. In Griffith's model, the interaction energy $U_{12}$ between an electron and the proton nucleus of the hydrogen atom amounts to [24],

$$
U_{12}=\mu_{0} \frac{\gamma_{g 1} \gamma_{g 2} e^{2}}{6 m_{1} m_{2}} \frac{\left(\boldsymbol{\sigma}_{\mathbf{1}} \cdot \boldsymbol{\sigma}_{2}\right)}{\pi d_{0}^{3}}=\frac{4}{6 c^{2}} \frac{e^{2}}{4 \pi \varepsilon_{0}} \frac{\gamma_{g 1} \gamma_{g 2}}{m_{1} m_{2}} \frac{\left(\boldsymbol{\sigma}_{\mathbf{1}} \cdot \boldsymbol{\sigma}_{2}\right)}{d_{0}^{3}}
$$


where the $\gamma^{\prime}$ 's are the gyromagnetic ratios, $m$ 's the rest masses and where $d_{0}$ is radius of the orbit. Like discussed before, see (46), the nuclear equivalent of the interaction can be obtained by replacing $e^{2} / 4 \pi \varepsilon_{0}$ by the nuclear equivalent $g \Phi_{0} / \lambda$.

Hence, from (69), we have for two identical particles,

$$
U_{12}=\frac{1}{c^{2}} \frac{4}{6} \frac{q_{e}^{2}}{4 \pi \varepsilon_{0}} \frac{\gamma^{2}}{m^{2}} \frac{\left(\boldsymbol{\sigma}_{1} \cdot \boldsymbol{\sigma}_{2}\right)}{d_{0}^{3}} \rightarrow \frac{1}{c^{2}} \frac{2}{3} \frac{g \Phi_{0}}{\lambda} \frac{\gamma^{2}}{m^{2}} \frac{\left(\boldsymbol{\sigma}_{1} \cdot \boldsymbol{\sigma}_{2}\right)}{d_{0}^{3}}=\frac{1}{c^{2}} \frac{2}{3}\left(\frac{\alpha \pi \hbar c}{2 d_{\min }^{\prime}}\right) \frac{\gamma^{2}}{m^{2}} \frac{\left(\boldsymbol{\sigma}_{1} \cdot \boldsymbol{\sigma}_{2}\right)}{d_{0}^{3}}
$$

The spins will align themselves in parallel or in anti-parallel, which gives, respectively,

$\boldsymbol{\sigma}_{1} \cdot \boldsymbol{\sigma}_{2}=\frac{\hbar^{2}}{4} \quad$ and $\quad \boldsymbol{\sigma}_{1} \cdot \boldsymbol{\sigma}_{2}=-\frac{3 \hbar^{2}}{4}$

Hence, the energy difference between the parallel spin condition and the antiparallel condition is given by

$$
\Delta E=\frac{1}{3 c^{2}}\left(\frac{\alpha \pi \hbar c}{d_{\min }^{\prime}}\right) \frac{\gamma^{2}}{m^{2}} \frac{\hbar^{2}}{d_{0}^{3}}
$$

Actually, the difference is $7 / 4$ times larger. This is due to an additional amount of energy as a consequence of the recoil of the bond in the higher state of energy. The correction factor can be found from the positronium case. For the positronium, we have from (71)

$$
\Delta E=\frac{1}{c^{2}} \frac{4}{6} \frac{q_{e}^{2}}{4 \pi \varepsilon_{0}} \frac{\gamma^{2}}{m^{2}} \frac{1}{d_{0}^{3}}=\frac{2 \gamma^{2}}{3 m c^{2}} \frac{g^{2} \hbar c}{d_{0}^{3}}
$$

Where the Bohr radius is given by,

$$
d_{0}=\frac{\hbar}{g^{2}(m / 2) c}
$$

Hence, from (74) and (75),

$$
\Delta E=\frac{1}{3} g^{8} m c^{2}
$$

Including the recoiling influence (beyond the scope of this article), the actual amount is $[25,26]$,

$$
\Delta E=\frac{1}{3} g^{8} m c^{2}+\frac{1}{4} g^{8} m c^{2}=\frac{7}{4}\left(\frac{1}{3} g^{8} m c^{2}\right) .
$$

Let us proceed by taking the recoil correction into account. Hence, from (77), 


$$
\Delta E=\frac{7}{12 c^{2}}\left(\frac{\alpha \pi \hbar c}{d_{\min }^{\prime}}\right) \frac{\gamma^{2}}{m^{2}} \frac{\hbar^{2}}{d_{\min }^{3}} \lambda^{3}
$$

The center of mass value of and the lab frame values of $\lambda$ have been shown in (65). Using this expression in (78) gives,

$$
\Delta E=\frac{7}{12 c^{2}}\left(\frac{\alpha \pi \hbar c}{d_{\min }^{\prime}}\right) \frac{\gamma^{2}}{m^{2}} \frac{\hbar^{2}}{d_{\min }^{3}}\left(\frac{2 m_{\pi}^{\prime} d_{\min }^{\prime}}{\alpha \pi(\hbar c)}\right)^{3}=\frac{14}{3} \frac{1}{(\alpha \pi)^{2}} \frac{\gamma_{g}^{2}}{d_{\min }^{\prime}} m_{\pi}^{\prime} \approx 4.65 m_{\pi}^{\prime} .
$$

Let us the define the mass $m_{q u}$ of the constituent mass as the mass of the quark if there were no interaction between the spins. With interaction, then we have

$$
m_{\pi}^{\prime}=2 m_{q u}^{\prime}-3 A m_{q u}^{\prime} \quad \text { and } m_{\rho}^{\prime}=2 m_{q u}^{\prime}+A m_{q u}^{\prime} .
$$

Hence $\Delta E=m_{\rho}^{\prime}-m_{\pi}^{\prime}=4 A m_{q u}^{\prime}$, and from (80),

$$
m_{q u}^{\prime}=\frac{m_{\pi}^{\prime}}{2-3 A} \rightarrow \Delta E=\frac{4 A}{2-3 A} m_{\pi}^{\prime} \text {. }
$$

From (79) and (81), we get $A=0.518 . m_{\pi}^{\prime}$.

This is surprisingly close to the empirical value $A=0.51 m_{\pi}^{\prime}$ as mentioned in Griffith's textbook. So, this empirical one has now got a theoretical basis. Because the mass $m_{K}^{\prime}$ of the kaon has got a theoretical basis as well, we may calculate the constituent mass $m_{s}^{\prime}$ of the strange quark $s$ from the following set of equations,

$$
\begin{aligned}
& 2 m_{u}^{\prime}-3 A_{m}^{\prime}=m_{\pi}^{\prime}=140 \mathrm{MeV} . \\
& m_{K}^{\prime}=m_{u}^{\prime}+m_{s}^{\prime}-3 \frac{m_{u}^{\prime 2}}{m_{u}^{\prime} m_{s}^{\prime}} A_{m}^{\prime}=3.57 m_{\pi}^{\prime}=489 \mathrm{MeV} \\
& A_{m}^{\prime}=0.51 m_{\pi}^{\prime}
\end{aligned}
$$

Solving this set for $m_{u}^{\prime}$ and $m_{s}^{\prime}$ reveals $m_{u}^{\prime}=300 \mathrm{MeV}$ and $m_{s}^{\prime}=478 \mathrm{MeV}$.

This means that the constituent masses of the quarks can be theoretically derived from a single reference for which we have adopted the constituent mass of the archetype quark. This mass of the constituent quark is the lab frame value of the weak boson interaction boson that binds the quark in the archetype meson. This implies that there is no reason to consider the quark flavors as elementary.

\section{The $Z$ boson}

There is somewhat more. Because the spin spin interaction is a boson phenomenon next to to the weak interaction, one may expect that, where the weak interaction manifests itself as 
the weak interaction boson, the spin spin interaction will manifest itself as a boson as well. The massive energy of this boson is the energy of $A$, which is a quarter of the energy of the mass difference between the pion and the rho. Hence, correcting this for the center of mass frame, we get

$$
\hbar \omega_{A}=\frac{775-140}{4} \cdot \frac{80.4}{140}=91.16 \mathrm{GeV} .
$$

This is just the value of the Z-boson. It is fair to conclude that the Z-boson is the manifestation of the spin spin interaction. Note that, while in the Standard Model the value of the $Z$ boson is empirically established, the spin spin interaction boson is actually determined by theory.

\section{The quark-scaling theorem}

The wave function of the simple pion model as shown in (53) is Schrodinger's wave equation, which in fact is the non-relativistic approximation of Dirac's covariant wave equation. In the non-relativistic approximation, Dirac's four-component wave equation falls apart into two two-component wave equations. The covariant format has been obtained from the covariant derivative as shown by (14). As is well known, Dirac adopted the Einsteinean energy formula as a starting point. He might have chosen Einstein's geodesic equation instead. There is no reason why the momenta in the geodesic equation would not allow the same momentum-wave function transformation as in the energy equation. But why doing so? The consideration is that the geodesic equation may give additional results on top of those from the energy equation. The reason is that it contains an additional symmetry: apart from energy conservation, it complies momentum conservation. In a previous study, I have shown that the simplicity of the one-dimensional meson model as described in the previous chapter allows a geodesic approach. Equating the results of both, yields a very interesting theorem. This theorem shows an invariance of the $\Phi_{0} / \lambda$ ratio,

$$
g \frac{\Phi_{0}}{\lambda}=\frac{\alpha \pi \hbar c}{4\left|k_{0}\right| d_{\min }^{\prime}}
$$

where $k_{0}=1 / 2$ and $d_{\text {min }}^{\prime}=0.853$ as shown in (56) and where $\alpha=0.69$. This ratio holds for all quarks. It means that the strength $\Phi_{0}$ as well as the range $\lambda^{-1}$ of the potential field may be different for different quark flavors under invariance of the of the $\Phi_{0} / \lambda$ ratio. As we shall see the quantities $k_{0}$ and $d_{\min }^{\prime}$ are subject to an almost negligible adaptation if the influence of the electromagnetic interaction between the quarks is taken into account. As mentioned before, the quoted numerical value has been initially established from an expression of the Gravitational Constant $G$ in terms of quantum mechanical quantities that could be derived by means of the geodesic view on Dirac's theory. The actual proof of this result is beyond the scope of this article, but the interested reader may find it in a previous article [21]. Nevertheless, the meaning of $\alpha$ can be understood from a simple consideration: it will be clear that the actual bond between the quark and the antiquark in a meson is 
sustained by the weak interaction boson. Hence, the spacing $2 d_{\min }=2 d_{\min }^{\prime} \lambda$ is expected about equal to a half wave length of the weak interaction boson $\hbar \omega_{W}$. Hence,

$\lambda=\frac{2\left(\hbar \omega_{W}\right) d_{\min }^{\prime}}{\alpha \pi(\hbar c)}$, where $\alpha$ is a dimensionless correction factor of order 1.

The difference from 1 is due to the fact that the weak interaction boson is a product of the far field determined by the far field boson and the strong interacting near field. Hence, the far field parameter $\lambda$ is only partly decisive. Neither this scaling theorem nor the concept of quarks as third-type Dirac particles are part of the canonic particle physics theory. Nevertheless they are a result of the application by sound theoretical principles. If we adopt that these results are complementary to the gauge based theory as summarized in the preceding chapter, there must be three modes for the boson interaction between the quark and the antiquark. If this boson interaction is visualized as a standing wave between the quarks, two of the modes can be conceived as transversal modes, it is tempting to conceive the third one as a longitudinal mode. As noted by Peskin and Schroeder[10, p.692] as well as in Griffiths textbook [1, p.367], the longitudinal mode has got no other explanation apart from a the mathematical one associated with the gauge invariance and the spontaneous breaking mechanism: the binding of particles that belong to the same $\mathrm{SU}(2)$ group, requires the existence of three independent boson fields. These three fields take up energy from an energetic background field, just by theoretical consequence. In the view described in this article a more physical interpretation is given. In this view, illustrated in figure 1, two of the boson fields are the transversal modes associated with the binding force due to the polarisable dipole and the third mode is due to the dipole created from the angular momenta, such as described in the previous chapter.

As explained in the previous chapter, the influence of the bare masses of the quarks in the energetic state of the pion is of negligible influence. Virtually, all energy is comprised by the binding energy due to the nuclear bosons. Under decay, the pion breaks up into W-bosons or photons, which will manifest themselves into a decay path of fermions. While the bosons fly at light speed, the fermions don't. Hence the energy of the fermions is the non-relativistic map of the energy from the ultra non-relativistic bosons. In principle the massive energy of these fermions can therefore be established by a relativistic correction of the boson energies.

Under recognition of the quark-scaling theorem, the mass generation mechanism can be well understood. Next to the scaling law (84) and the weak interaction boson law (85), the only other one needed is the quantum mechanical oscillator law,

$$
\frac{m_{m} \omega^{2}}{2}=g \Phi_{0} k_{2} \lambda^{2}
$$

Conventionally, $m_{m}$ represents is the central mass of the oscillator. In the relativistic model described in chapter 4, it does not represent the individual bare masses of the two bodies, but it is an equivalent mass that captures the energy of the field. As usual, $\omega$ is related with the vibration energy $E_{n}=(n+1 / 2) \hbar \omega$. Considering (53), we may state, 
$\hbar \omega=\hbar \omega_{W}$.

Hence, from (86) and (87),

$\Phi_{0}=\frac{m_{m}^{\prime}}{8 g k_{2} d_{\min }^{\prime 2}}$

Invoking (84) on this gives,

$m_{m}^{\prime}=m_{m} c^{2}=\frac{8 g\left|k_{0}\right| d_{\min }^{\prime 2} k_{2}\left(\hbar \omega_{W}\right)}{\left(k_{0} \alpha \pi\right)^{2}}$

This represents the relativistic mass of the pion. Because of the required non-relativistic correction, this expression cannot serve to calculate the lab frame value of the meson's mass. Nevertheless, the expression is very relevant, because it expresses that mass ratios can be calculated as ratios of $k_{2}\left(d^{\prime}\right)$. Taking the mass value of the pion in the lab frame as the reference, the meson's mass spectrum can be established from the behavior of $k_{2}\left(d^{\prime}\right)$.

As noted before, the scaling theorem and the quark description as a third type Dirac particle are not part of canonic particle physics theory. In my previous studies it has been shown that the scaling theorem can be successfully applied for calculating the mass spectrum of mesons and baryons. On top of that, we have already concluded in chapter 5 that the constituent masses of the quark flavors can be calculated as well, thereby revealing that these masses are scaled values of the archetype quark. This means that the quark flavors strange $(s)$, charm $(c)$ and bottom $(b)$ should not be regarded as elementary particles, but instead scaled versions of the up/down quark archetype $(u / d)$.

\section{The Higgs boson}

In the preceding chapters it has been demonstrated that the meson's mass spectrum can be explained from the quark's far field as defined in (50), supplemented by a near field from a dipole moment. The far field is a scalar field obtained from the steady state solution of a Proca-type wave equation with the format

$\frac{1}{c^{2}} \frac{\partial^{2} r \Phi}{\partial t^{2}}-\frac{\partial^{2}}{\partial r^{2}} r \Phi+\lambda^{2} r \Phi=\rho_{H}(r, t)$

where $\rho_{H}(r, t)$ is a Dirac-type pointlike source that can be expressed as,

$\rho_{H}(r, t)=4 \pi \lambda \Phi_{0} \delta(r) H(t)$,

where $H(t)$ is Heaviside's step function. Its solution is given by [20], 
$r \Phi(r, t) \leftrightarrow \frac{\Phi_{0}}{\lambda} \frac{1}{s} \exp \left[-\left(\lambda r \sqrt{\left.s^{2} /(\lambda c)^{2}+1\right)}\right]\right.$

If, under violence of particle collisions, the equilibrium between the quarks is broken, the far field bosons will show up in decay channels of pairs of gamma photons, W-bosons or Zbosons, which will manifest themselves into a decay path of fermions. Momenta and energies of these fermions can be measured and can be traced back to numerical values for the energy of a nuclear boson pair. So, ultimately, the Higgs field will show up as two quantum fields, instead of the single one that is expected by the Standard Model. The massive energy of the far field part, if interpreted as a single boson, would therefore be assigned as,

$m_{H}^{\prime} \approx 2 \lambda(\hbar c)$.

Subsequent application of (85) on this gives,

$m_{H}^{\prime}=\frac{4 d_{\min }^{\prime} m_{W}^{\prime}}{\alpha \pi}=127 \mathrm{GeV}$

which nicely fits with experimental evidence from the detection in 2012 by CERN of a 126.5 GeV bosonic particle.

One might wonder about the factor 2. The Standard Model value $m_{H}^{\prime \prime \prime}$ of the Higgs boson in natural units documented in [10] gives, under consideration of (48),

$m_{H}^{\prime \prime \prime}=\mu_{H} \sqrt{2} \approx 2 \lambda \rightarrow m_{H}^{\prime}=2 \lambda(\hbar c)$.

This is just the same as (91). The result gives a strong support to the viability as developed in this article, including its compatibility with the Standard Model. However, unlike as in the Standard Model, the numerical value is now established by theory.

\section{Electric charge and electromagnetic interaction}

In chapter 3, it has been shown that the weak interaction boson emitted by a quark is subject to weak isospin, where the weak isospin vector, although closely related, is somewhat different from the Pauli vector. The eigen value of the weak isospin vector is $I_{z}= \pm 1 / 2$. In that respect, there is no difference with the Pauli-type eigen value of the second dipole moment of the quark, which we have denoted as isospin. Where in the Standard Model the weak isospin is adopted as an axiomatic attribute of the archetype, it is in our model a straightforward consequence of a third-type Dirac particle. In table II it has been suggested that the Pauli-type isospin states of the quarks can be related with the electric charge state of the meson. Where in our model, the $u$ and $d$ quarks are identical, albeit in a different state of isospin, they are different in the Standard Model because of the hypercharge bias $\mathrm{Y}$ and defined opposite values of the weak isospin state. As long as the 
charge of the meson is considered as a holistic quantity, the hypercharge bias does not make any difference. Its role will be discussed later in this article.

\begin{tabular}{|l|l|l|l|l|l|l|l|}
\hline meson & $\begin{array}{l}\text { isospin } \\
\text { modes }\end{array}$ & recode & $\mathrm{Q}$ & $\begin{array}{l}\text { pseudo } \\
\text { scalar }\end{array}$ & vector & $I_{z}$ & $Y$ \\
\hline \multirow{4}{*}{$u \bar{u}$} & $\uparrow \uparrow$ & $u \bar{d}$ & 1 & $\pi^{+}$ & $\rho^{+}$ & $1 / 2+1 / 2$ & $(1 / 3-1 / 3) / 2$ \\
\cline { 2 - 8 } & $\downarrow \downarrow$ & $\bar{u} d$ & -1 & $\pi^{-}$ & $\rho^{-}$ & $-1 / 2-1 / 2$ & $(-1 / 3+1 / 3) / 2$ \\
\cline { 2 - 8 } & \multirow{3}{*}{\begin{tabular}{l}
$\uparrow \downarrow \pm \downarrow \uparrow$ \\
\cline { 2 - 7 }
\end{tabular}} & $(u \bar{u}-\bar{d} d) / \sqrt{2}$ & 0 & $\pi^{0}$ & $\rho^{0}$ & 0 & 0 \\
\cline { 2 - 7 } & $(u \bar{u}+\bar{d} d) / \sqrt{2}$ & 0 & $\mathrm{x}$ & $\omega$ & 0 & 0 \\
\hline
\end{tabular}

In the analysis so far presented in this article, the electromagnetic interaction has been regarded as a second order effect. In this chapter, it will be shown that the scaling theorem applied on quarks conceived as third type Dirac particles is a powerful instrument for calculating the mass difference between the charged pion and the neutral pion. The method how to do it under use of the scaling theorem alone has been documented before [20]. In this chapter an update will be given, which takes the particular characteristic of the third type Dirac into account. The task to be done is including the electromagnetic interaction into account as an additional force on the nuclear force between the quark and the antiquark. We may combine this additional force with the weak interaction force, implying that each quark feels a repulsive force $F(r)$ from the other quark, such that

$$
F(r)=-g \frac{\mathrm{d} \Phi}{\mathrm{d} r} \pm p \frac{e^{2}}{4 \pi \varepsilon_{0} r^{2}}
$$

where $p$ is a dimensionless factor, which depends on the composition of the pion. If we would suppose that the quark and the antiquark are electrically symmetrical, like done [20], we would adhere a positive or a negative value $e / 2$ to each of the quarks. In that case, the $p$ value would be $+1 / 4$ for the charged pions and $-1 / 4$ for the neutral pion. In the canonical model with unequal charges for the $u$ quark and the $d$ quark we have $p=2 / 9$ for charged pions and $p=-2 / 9$ for neutral pions.

Under the unification condition

$e^{2}=4 \pi \varepsilon_{0} \hbar c g^{2}$

we get for (94)

$$
F(r)=-g \frac{\mathrm{d} \Phi}{\mathrm{d} r} \pm p \frac{g^{2} \hbar c}{r^{2}}
$$

This means that the far field potential is modified due to electric interaction into, 


$$
F(r)=\frac{\Phi_{0}}{\lambda r} \exp (-\lambda r) \pm p \frac{g \lambda \hbar c}{\lambda r}
$$

Now, the potential $\Phi\left(x^{\prime}\right)$ of the field built up by the quarks felt by the center of mass, expanded along the dipole axis, is built up by the near field $\Phi_{N}\left(x^{\prime}\right)$ from the dipole moment, the far field $\Phi_{F}\left(x^{\prime}\right)$ component of the weak interaction and the electromagnetic potential $\Phi_{e m}\left(x^{\prime}\right)$, such that

$$
\begin{aligned}
& \Phi\left(x^{\prime}\right)=\Phi_{N}\left(x^{\prime}\right)-\Phi_{F}\left(x^{\prime}\right) \pm \Phi_{e m}\left(x^{\prime}\right) \text { with } x^{\prime}=\lambda x, \\
& \Phi_{N}\left(x^{\prime}\right)-\Phi_{F}\left(x^{\prime}\right)=\Phi_{0}\left(k_{0}+k_{2} x^{\prime 2}+\ldots . .\right), \\
& \Phi_{e m}\left(x^{\prime}\right)=w \frac{1}{4} \frac{g(\hbar c) \lambda}{d^{\prime}}\left(2+\frac{2}{d^{\prime 2}} x^{\prime 2}+\ldots . .\right) ; d^{\prime}=d \lambda .
\end{aligned}
$$

Note: $\Phi_{e m}^{\prime}$ holds under the assumption of equal charge distribution over the two quarks. The dimensionless factor $w$ is the one introduced in (49) for weighing the far field relative to the near field. As discussed, this factor is close to 2 . This factor has not been taken into account in my previous study [20] on the electromagnetic interaction.

Eq. (98) can now be written as,

$$
\begin{aligned}
& \left.\Phi\left(x^{\prime}\right)=\Phi_{0}\left(k_{0}^{\prime}+k_{2}^{\prime}\right) x^{\prime 2}+\ldots . .\right), \text { with } \\
& k_{0}^{\prime}=k_{0} \pm \frac{1}{2} w \frac{g(\hbar c) \lambda}{d^{\prime} \Phi_{0}} ; \quad k_{2}^{\prime}=k_{2} \pm \frac{1}{2} w \frac{g(\hbar c) \lambda}{d^{\prime} \Phi_{0}} .
\end{aligned}
$$

Hence, after invoking the invariance $\Phi_{0} / \lambda$ as expressed by (84),

$$
k_{0}^{\prime}=k_{0} \pm w \frac{g^{2} d_{\min }^{\prime}}{\alpha \pi d^{\prime}} ; \quad k_{2}^{\prime}=k_{2} \pm w \frac{g^{2} d_{\min }^{\prime}}{\alpha \pi d^{\prime}}
$$

The potential is minimum if $k_{0}^{\prime}$ is minimum. Hence, the minimum values for $k_{0}^{\prime}$ and the spacing values for minimum potential are slightly different from $k_{0}\left(d_{\min }^{\prime}\right)=1 / 2$ and $d_{\text {min }}^{\prime}=0.852$ as they were calculated without the electromagnetic interaction. As a consequence of the shift, the mass formula (89) has to be modified into,

$$
m_{m}^{\prime}=m_{m} c^{2}=\frac{8 g\left|k_{0}\right| d_{\min }^{\prime 2} k_{2}^{\prime}\left(\hbar \omega_{W}\right)}{\left(k_{0}^{\prime} \alpha \pi\right)^{2}}
$$

The reason for the difference between $k_{0}$ and $k_{0}^{\prime}$ in, respectively, the numerator and denominator of (101), has been discussed in my previous study [20]. The expression reveals two different mechanisms that influence the mass difference due to electromagnetic 
interaction. One of these is the change of the binding energy due to $k_{0}^{\prime}$ and the other one is the change in harmonic oscillator ground state energy due to $k_{2}^{\prime}$. The effects are opposite. As shown in [20], it explains the rather queer phenomenon that, while charged pions heve a larger mass value than neutral pions, the opposite is true for kaons. As far as I know, similar detailed successful calculations have not been obtained so far with Standard Model theory. It is the quark scaling theorem (84), which have no equivalent in the Standard Model, that reveals such subtleties. My previous study contains rather ore extensive details for calculation, including documentation of numerical programs in Wolfram's Mathematica [22]. In that study, the calculated mass difference amounts to $2.3 \mathrm{MeV}$, which is just half of the empirical value. Only recently, I realized myself that the factor 2 difference is due to the $w$ factor in (100), for which, at that time, I had no explanation.

\section{Baryons}

Where a meson can be conceived as the one-body equivalent of a two-body harmonic oscillator, a baryon can be conceived as the one-body equivalent of a three-body harmonic oscillator. The one-body equivalent of the three-body quantum mechanical oscillator can be analyzed in terms of pseudo-spherical Smith Whitten coordinates [27]. The Smith-Whitten system of coordinates is six-dimensional. Next to a (hyper)radius $\rho$, the square of which is the sum of the squared spacings between three bodies, there are five angles $\varphi, \vartheta, \alpha, \beta, \gamma$, where $\varphi$ and $\vartheta$ model the changes of shape of the triangular structure and where $\alpha, \beta$ and $\gamma$ are the Euler angles. The latter ones define the orientation of the body plane in 3D-space. The planar forces between three identical interacting bodies are not only the cause of dynamic deformations of the equilateral structure, but are also the cause of a Coriolis effect that result in vibra-rotations around the principal axes of inertia of the three-body structure [28]. The application of this approach for baryons has been documented by the author in [29], showing that the wave equation of the quasi-equilateral baryon structure can be formulated as

$$
-\alpha_{0}\left\{\frac{d^{2} \psi}{d \rho^{\prime 2}}+\frac{5}{\rho^{\prime}} \frac{d \psi}{d \rho^{\prime}}+\frac{R(m, v, k)}{\rho^{\prime 2}} \psi\right\}+V^{\prime}\left(\rho^{\prime}\right)=E^{\prime} \psi,
$$

where $\alpha_{0}=\frac{\hbar^{2} \lambda^{2}}{6 m g \Phi_{0}} ; E^{\prime}=\frac{E}{3 g \Phi_{0}} ; V^{\prime}=\frac{V}{3 g \Phi_{0}} ; \rho^{\prime}=\rho \lambda$, and

$$
\begin{aligned}
& V\left(\rho^{\prime}\right)=3 g \Phi_{0}\left(k_{0}+k_{2} \rho^{\prime 2}+\ldots \ldots\right) \\
& R(m, v, k)=4 m+|v-k|(4 m+|v-k|+4)
\end{aligned}
$$

This wave function is the three-body equivalent of the pion's two-body wave equation shown in (5-3). In the ground state $m=0$. Hence,

$$
R=R(0, v, k)=l(l+4) ; l=|v-k| .
$$


The radial variable $\rho$ is the already mentioned hyper radius. The potential field is just the threefold of the potential field in the wave equation of the pion. There are three quantum numbers involved. Two of those are left in the ground state, effectively bundled to a single one. The quantum number $k$ allows a visual interpretation, while $v$ is difficult to visualize. The impact of $k$ is shown in figure 6 . It illustrates the motion of the center of mass under influence of $k$. Note that this rotation is quite different from a rotation of the triangular frame around the center of mass. It is the center of mass itself that rotates, while the frame does not. Actually, the small motions of the individual quarks are responsible for this motion.

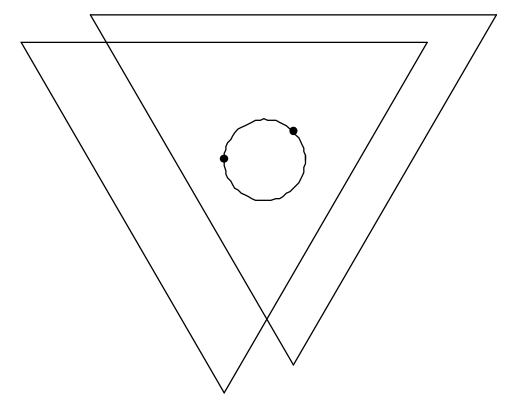

Figure 6: . Physical interpretation of the motion associated with the angular quantum number $k$.

As shown in [29], this relatively simple wave function expression allows a pretty accurate calculation of the mass spectrum of baryons. The octet states in the baryon classification are the counter part of the meson pseudoscalar states, the decuplet states are the counter part of the vector mesons. A single integer step in the quantum number $l$, brings the $p, n / \Delta$ level to the $\Sigma / \Sigma^{*}$-level, etc. The results of the mass calculations are shown in the right-hand part of the tables III and IV.

Table I: Re-interpretation of the light baryon octet

\begin{tabular}{|c|c|c|c|c|c|c|c|c|c|}
\hline & \multicolumn{9}{|c|}{ singlet states (octet) } \\
\hline baryon & modes & code & recode & $\begin{array}{l}\text { spin } \\
\text { (nuclear) }\end{array}$ & bias & charge & symb & $\begin{array}{l}\text { mass } \\
\text { calc. }\end{array}$ & $\begin{array}{l}\text { mass } \\
\text { act. }\end{array}$ \\
\hline \multirow[t]{2}{*}{$(u u) \bar{u}$} & $(\uparrow \downarrow) \uparrow$ & $u d \bar{u}$ & udu & $+1 / 2$ & $+1 / 2$ & 0 & $\mathrm{p}$ & 934 & 938 \\
\hline & $(\uparrow \downarrow) \downarrow$ & $u d \bar{d}$ & udd & $-1 / 2$ & $+1 / 2$ & & $\mathrm{n}$ & 934 & 939 \\
\hline$(u u) \bar{s}$ & $(\uparrow \downarrow) \uparrow$ & $u d \bar{s}$ & uds & $-1 / 2$ & $+1 / 2$ & 0 & $\Lambda^{0}$ & 1105 & 1115 \\
\hline \multirow[t]{3}{*}{$(u \bar{u}) s$} & $(\uparrow \uparrow) \downarrow$ & $u \bar{u} s$ & uus & $+1 / 2$ & $+1 / 2$ & 1 & $\Sigma^{+}$ & 1170 & 1189 \\
\hline & $(\downarrow \downarrow) \downarrow$ & $d \bar{d} s$ & dds & $-3 / 2$ & $+1 / 2$ & -1 & $\Sigma^{-}$ & 1170 & 1197 \\
\hline & $(\uparrow \downarrow) \downarrow$ & $u \bar{d} s$ & uds & $-1 / 2$ & $+1 / 2$ & 0 & $\Sigma^{0}$ & 1170 & 1192 \\
\hline$(s s) \bar{u}$ & & \multicolumn{8}{|c|}{ spatial spin conflict } \\
\hline \multirow[t]{2}{*}{$(s \bar{s}) u$} & $(\downarrow \uparrow) \uparrow$ & $s \bar{s} u$ & ssu & $-1 / 2$ & $+1 / 2$ & 0 & $\Xi^{0}$ & 1314 & 1321 \\
\hline & $(\downarrow \uparrow) \downarrow$ & $s \bar{s} d$ & ssd & $-3 / 2$ & $+1 / 2$ & -1 & $\Xi^{-}$ & 1314 & 1314 \\
\hline$(s s) \bar{s}$ & & \multicolumn{8}{|c|}{ spatial spin conflict in singlet state } \\
\hline
\end{tabular}


Table II: Re-interpretation of the light baryon decuplet

\begin{tabular}{|c|c|c|c|c|c|c|c|c|c|}
\hline & \multicolumn{9}{|c|}{ triplet states (decuplet) } \\
\hline baryon & modes & & recode & $\begin{array}{l}\text { spin } \\
\text { (nuclear) }\end{array}$ & bias & charge & symb & $\begin{array}{l}\text { mass } \\
\text { calc. }\end{array}$ & $\begin{array}{l}\text { mass } \\
\text { act. }\end{array}$ \\
\hline \multirow[t]{4}{*}{$(u \bar{u}) u$} & $(\uparrow \uparrow) \uparrow$ & $u \bar{u} u$ & udu & $+1 / 2$ & $+1 / 2$ & 1 & $\Delta^{+}$ & 1237 & 1232 \\
\hline & $(\downarrow \downarrow) \downarrow$ & $d \bar{d} d$ & udd & $-1 / 2$ & $+1 / 2$ & 0 & $\Delta^{0}$ & 1237 & 1232 \\
\hline & $(\uparrow \downarrow) \uparrow$ & $u \bar{d} u$ & uuu & $+3 / 2$ & $+1 / 2$ & 2 & $\Delta^{++}$ & 1237 & 1232 \\
\hline & $(\downarrow \uparrow) \downarrow$ & $d \bar{u} d$ & ddd & $-3 / 2$ & $+1 / 2$ & -1 & $\Delta^{-}$ & 1237 & 1232 \\
\hline$(u u) \bar{s}$ & & \multicolumn{8}{|c|}{ not possible in triplet state } \\
\hline \multirow[t]{3}{*}{$(u \bar{u}) s$} & $(\uparrow \downarrow) \downarrow$ & $u \bar{d} s$ & uus & $+1 / 2$ & $+1 / 2$ & 1 & $\Sigma^{*+}$ & 1377 & 1382 \\
\hline & $(\downarrow \uparrow) \downarrow$ & $d \bar{u} s$ & dds & $-3 / 2$ & $+1 / 2$ & -1 & $\Sigma^{*-}$ & 1377 & 1387 \\
\hline & $(\downarrow \downarrow) \downarrow$ & $(d \bar{d} s)$ & uds & $-1 / 2$ & $+1 / 2$ & 0 & $\Sigma^{* 0}$ & 1377 & 1383 \\
\hline$(s s) \bar{u}$ & & \multicolumn{8}{|c|}{ not possible in triplet state } \\
\hline \multirow[t]{2}{*}{$(s \bar{s}) u$} & $(\downarrow \uparrow) \uparrow$ & $s \bar{s} u$ & ssu & $-1 / 2$ & $+1 / 2$ & 0 & $\Xi^{* 0}$ & 1521 & 1531 \\
\hline & $(\downarrow \uparrow) \downarrow$ & $s \bar{s} d$ & ssd & $-3 / 2$ & $+1 / 2$ & -1 & $\Xi^{*-}$ & 1521 & 1536 \\
\hline$(s \bar{s}) S$ & $(\downarrow \uparrow) \downarrow$ & $S \bar{S} S$ & SSS & $-3 / 2$ & $+1 / 2$ & -1 & $\Omega^{-}$ & 1671 & 1672 \\
\hline
\end{tabular}

The most left columns of the table need some explanation. To avoid spatial spin conflicts with Pauli's law, in this modeling of baryons as a planar three-body configuration one of the three quarks should be different from the other two. A different state of isospin makes a quark not more or not less different from an identical other one as a different state of "temporal spin" that turns a into an antiquark. Hence representing a different state of isospin as an antiparticle like shown in the tables is an allowable way of coding. Curiously, the coding of isospin state similar as the coding in terms of two particles next to an antiparticle does not make any difference for the classification. This phenomenon has been mentioned in the context of mesons as well (see chapter 4).Similarly as in the case of mesons, $u$ and $d$ quarks are considered as identical particles in a different state of nuclear spin. Unlike as in the case of mesons, baryons have some charge bias. Similarly as in the case of mesons, the scaled quarks $s$ (and higher) have a only a single state of spin. Note the difference between the $u$ and $d$ coding with the $u$ and $\mathrm{d}$ (re)coding.

Where the mesons in this article are considered as members of an $\mathrm{SU}(2)$ group, it makes sense considering the baryons as members of an $\mathrm{SU}(3)$ group. This is most obvious if one the three quarks would be in antiparticle state. This is reflected in the wave function representation shown in (104). Because the archetype quarks are supposed to be identical, they hold each other in equilibrium by spatial momenta $\left(p_{x}, p_{y}\right)$ with relative values of, respectively, $(\sqrt{3} / 2,1 / 2),(-\sqrt{3} / 2,1 / 2)$ and $(0,1)$. These values are reflected in the spatial components of the wave function. While two of the quarks can be in particle state with opposite direction of their spatial spin, the third one has to be in antiparticle state. 
$\Psi_{p a}=\left[\begin{array}{lll}\Psi_{1 t} & \Psi_{1 x} & \Psi_{1 y} \\ \Psi_{2 t} & \Psi_{2 x} & \Psi_{2 y} \\ \Psi_{3 t} & \Psi_{3 x} & \Psi_{3 y}\end{array}\right] \rightarrow\left[\begin{array}{ccc}-\mathrm{i} b & a \sqrt{3} / 2 & a / 2 \\ b & -\mathrm{i} a \sqrt{3} / 2 & \mathrm{i} a / 2 \\ -\mathrm{i} b & 0 & -a\end{array}\right]$

It is not difficult to prove that, under proper scaling of the amplitudes, this matrix is unitary (i.e. $\Psi \Psi^{+}=1$, where $\Psi^{+}$is the transpose conjugate of $\Psi$ ) and that its determinant is equal to 1 for any value of the ratio $a / b$. In a conservative field of forces, like it is the case of interaction between the quarks as a consequence of their nuclear potential fields, the ratio $a / b$ is subject to change. This implies that the nine-component spinor $\Psi_{a m p}$ may rotate over eight spatial angles $\bar{\vartheta}\left(\vartheta_{i}\right)$ in a nine-dimensional spinor space. This rotation is the equivalence of the weak interaction in the meson case. The bosons involved in SU(3) are known as gluons. Because the wave function shown in (102) is a single dimensional center of frame Schrodinger approximation of the generic nine-dimensional wave function (104), the fine nuances have disappeared. But, in fact, there is no conflict here with the Standard Model.

\section{Discussion and conclusion}

In this article it has been shown how two unrecognized theoretical consequences from Dirac's electron theory may influence the view on the Standard Model of particle physics without substantially affecting its basics of SU(2) and SU(3) gauging, electroweak unification and the mass generation mechanism from the Higgs field. Most of the presented results have been documented in literature by me before in more detail, some in journals, others in prepublications that met opposition because of a seeming conflict with common views that are considered as proven in the wealth of studies and experiments in the high standard of present theory. The highlight on two additional principles that are not yet covered in the Standard Model, may help showing that results obtained before might be useful complements to the present status. This holds in particular for the mass calculations, because, while mass, next to charge, is the main attribute of physical particles, its assessment in the Standard Model is mainly empirically based and has not yet reached the same high standard as many other attributes. The two basic principles highlighted in this article that can be added to the Standard Model are,

1. The quark is an unrecognized Dirac particle that has, next to the well-known real dipole moment associated with the elementary angular momentum $\boldsymbol{h}$, a second real dipole moment associated with an elementary linear dipole $h / c$, which, unlike as in the case of electrons, is polarisable in a scalar potential field.

2. Deriving Dirac's fermionic wave equation from Einstein's geodesic equation rather than from Einstein's energy expression reveals a complementary property to the quark conceived as a Dirac particle described in the first highlight. This property is the invariance of the frame-independent ratio $\Phi_{0} / \lambda$, where $\Phi_{0}$, expressed in units of energy, is a measure of the quark's potential and where $\lambda$, expressed in $\mathrm{m}^{-1}$, is a measure for the range of the quark's potential. In the article, this property is dubbed as the quark-scaling theorem. 
The details of the derivation of these two principles can be found in, respectively, [7] and [21]. In the theory reviewed in this article, the quark is an energetic pointlike particle that emits energy into the vacuum filled with background energy. The symmetry of the quark's potential due to the background energy is broken. In the view developed in this article, the break is due to the polarisable dipole moment. If the weak interaction and electromagnetism have a common source split up because of a spontaneous break event in the background field, the polarisable dipole might have turned off from an electron-type imaginary one into a real one as a result of the very same event. In that respect there is no principal difference with the Standard Model. The mass generation mechanism remains the same with the difference that, under recognition of the quark-scaling mechanism, the mass of hadrons can be traced back to the massive energy evoked as the ground state energy of the archetype meson. As a consequence, the number of elementary particles is substantially reduced. It has been shown that the hadron masses can be derived from a single reference for which we have adopted the rest mass of the pion The mass value of the Z-boson and the mass value of the Higgs boson, which in present theory both are empirically assessed, have been related by theory with the mass value of weak interaction, which is shown to be the non-relativistic equivalent of the lab frame mass value of the pion. Although the view outlined in this article is shown being compatible with $\mathrm{SU}(2)$ and $\mathrm{SU}(3)$ gauging as well as with electroweak unification, the view on electric charge is slightly different. Where in the Standard Model electric charge is seen as an attribute of an individual quark, the adopted view in this article is considering electric charge as a holistic attribute of an hadron, evoked as a result from the spin characteristics of all dipole momenta involved in the hadron. Similarly as mass is a holistic result in which bare masses are of second order, electric charge might be a holistic result as well with no need to adopt the usual 1/3-2/3 split.

The theory described in this article is a structural view on particle physics with a physical interpretation on some of the axiomatic principles adopted in the mathematical formalism of the Standard Model. The model description of the mesons and the baryons is based upon a non-relativistic approximation of the multi-dimensional Dirac's fermionic wave equation to a single dimensional Schrödinger one in the center of mass frame of hadrons, extended by separate additions of some second order effects not covered in the approximation and interpreted in the lab frame after relativistic correction. Hence, it lacks the rigidity of the conventional Standard Model description. On the other hand, the recognition and inclusion of the quark's polarisable dipole moment and the quark-scaling theorem reveals results that are not obtained so far in the present state of theory.

\section{References}

[1] D. Griffiths, Introduction to Elementary Particles, ISBN 3527406018, Wiley (2008)

[2] P.A.M. Dirac, Proc.Royal Soc. London, A 117, 610 (1928)

[3] J.D. Bjorken, S.D. Drell, Relativistic Quantum Mechanics, McGraw-Hill Book Cie (1964)

[4] T. Nakano, N. Nishjima, Pogr. Theor. Phys. 10 (5), 581, doi:10.1143/PTP.10.581 (1953)

[5] M. Gell-Mann, II Nuovo Cmento 4(S2), 848, doi:10.1007/BF02748000 (1956)

[6] M. Gell-Mann and Y. Ne-eman, The Eightfold Way: A Review, A.A. Benjamin Inc. NewYork-Amsterdam , 168 (1964) 
[7] E. Roza, preprints.org, doi:10.20944/preprints202002.0275.v1(2020), submitted to Foundations of Physics.

[8] D. Perkins, Introduction to High Energy Physics, $4^{\text {th }}$ Ed., Cambridge Univ. Press, Cambridge UK (2000)

[9] C.N. Yang, R. Mills, Phys. Rev. 96, 191, 1954

[10] M.E. Peskin , D.V. .Schroeder, An introduction to Quantum Field Theory, Addison-Wesley (1995)

[11] A. Salam, J.C. Ward, Nuovo Cimento 11 (4), 568, doi:10.1007/BF02726525 (1959)

[12] S. Weinberg, Phys. Rev. Lett 19 (21), 1264, doi:10.1103/PhysRevLett.191264W (1967)

[13] P. Debye and E. Huckel, Physik. Zeitschrift, vol. 24, 9, 185 (1923)

[14] P. Higgs, Phys. Rev. Lett. 13 (16 ), 509 (1964)

[15] F. Englert, R. Brout, Phys. Rev. Lett. 13 (9), 321 (1964)

[16] J Goldstone, Nuovo Cimento, 19, 154, doi:10.1007/BF02812722 (1961)

[17] J Goldstone, A. Salam, S. Weinberg, Phys. Rev. 127, 965, doi:10.1103/PhysRev.127.965 (1962)

[18] G. Zweig in "Developments In The Quark Theory Of Hadrons", Vol. 1*, 22-101 and CERN Geneva - TH. 401 (1964).

[19] F. Wilczek, Nature (London), 445, 156 (2007)

[20] E. Roza, Phys. Essays, 25, 2 (2012)

[21] E. Roza, Results in Phys., 6, 149 (2016)

[22] S. Wolfram, Mathematica (Addison-Wesley Publishing Inc. Redwood City, Ca (1991

[23] E. Roza, doi:10.20944/preprints201701.0076.v2 (2019)

[24[ D.J. Griffiths, Am. J. Phys. 50, 698 (1982)

[25] C. Cohen-Tannoudji, B. Diu, Quantum Mechanics, Hermann, Paris (1977)

[26] A. Czarnecki, K. Melnikov, A. Yelkovsky, Phys. Rev. Letters, 82, 311 (1999)

[27] R.C. Whitten and F.T. Smith, J. Math. Phys. 9, 1103 (1968)

[28]P. Bartlett, B.J. Howard, Molecular Physics, Vol. 70, No. 6, 1001 (1990)

[29] E. Roza, Phys. Essays, 27, 3, 390 (2014) 
